OPEN ACCESS

Edited by: Filippo Caraci,

University of Catania, Italy

Reviewed by:

Rosalia Crupi,

University of Messina, Italy

Raffaella Gozzelino,

New University of Lisbon, Portugal

*Correspondence:

Michela Rosini

michela.rosini@unibo.it

Cristina Lann

cristina.lanni@unipv.it

${ }^{\dagger}$ These authors have contributed equally to this work

Specialty section:

This article was submitted to Experimental Pharmacology and Drug Discovery,

a section of the journal

Frontiers in Pharmacology

Received: 18 June 2020

Accepted: 30 July 2020

Published: 14 August 2020

Citation:

Fagiani F, Catanzaro M, Buoso E, Basagni F, Di Marino D, Raniolo S,

Amadio M, Frost EH, Corsini E, Racchi M, Fulop T, Govoni S, Rosini M and Lanni C (2020) Targeting Cytokine Release Through the Differential Modulation of Nrf2 and NF- $\kappa B$ Pathways by Electrophilic/NonElectrophilic Compounds.

Front. Pharmacol. 11:1256. doi: 10.3389/fphar.2020.01256

\section{Targeting Cytokine} Release Through the Differential Modulation of Nrf2 and NF-KB Pathways by Electrophilic/ Non-Electrophilic Compounds

Francesca Fagiani ${ }^{1,2 \dagger}$, Michele Catanzaro ${ }^{1 \dagger}$, Erica Buoso ${ }^{1}$, Filippo Basagni ${ }^{3}$, Daniele Di Marino ${ }^{4}$, Stefano Raniolo ${ }^{5}$, Marialaura Amadio ${ }^{1}$, Eric H. Frost ${ }^{6}$, Emanuela Corsini ${ }^{7}$, Marco Racchi ${ }^{1}$, Tamas Fulop ${ }^{8}$, Stefano Govoni ${ }^{1}$, Michela Rosini ${ }^{3 *}$ and Cristina Lanni ${ }^{1 *}$

\begin{abstract}
${ }^{1}$ Department of Drug Sciences, Pharmacology Section, University of Pavia, Pavia, Italy, ${ }^{2}$ Scuola Universitaria Superiore IUSS Pavia, Pavia, Italy, ${ }^{3}$ Department of Pharmacy and Biotechnology, University of Bologna, Bologna, Italy, ${ }^{4}$ Department of Life and Environmental Sciences, New York-Marche Structural Biology Center (NY-MaSBiC), Polytechnic University of Marche, Ancona, Italy, 5 Università della Svizzera Italiana (USI), Faculty of Biomedical Sciences, Institute of Computational ScienceCenter for Computational Medicine in Cardiology, CH-Lugano, Switzerland, ${ }^{6}$ Department of Microbiology and Infectiology, Centre de Recherches Cliniques, Faculty of Medicine and Health Sciences, University of Sherbrooke, Sherbrooke, QC, Canada, ${ }^{7}$ Department of Environmental Science and Policy, Università degli Studi di Milano, Milan, Italy, ${ }^{8}$ Geriatric Division, Department of Medicine, Faculty of Medicine and Health Sciences, Research Center on Aging, University of Sherbrooke, Sherbrooke, QC, Canada
\end{abstract}

The transcription factor Nrf2 coordinates a multifaceted response to various forms of stress and to inflammatory processes, maintaining a homeostatic intracellular environment. Nrf2 anti-inflammatory activity has been related to the crosstalk with the transcription factor NF- $\mathrm{kB}$, a pivotal mediator of inflammatory responses and of multiple aspects of innate and adaptative immune functions. However, the underlying molecular basis has not been completely clarified. By combining into new chemical entities, the hydroxycinnamoyl motif from curcumin and the allyl mercaptan moiety of garlic organosulfur compounds, we tested a set of molecules, carrying (pro)electrophilic features responsible for the activation of the Nrf2 pathway, as valuable pharmacologic tools to dissect the mechanistic connection between Nrf2 and NF-KB. We investigated whether the activation of the Nrf2 pathway by (pro)electrophilic compounds may interfere with the secretion of pro-inflammatory cytokines, during immune stimulation, in a human immortalized monocyte-like cell line (THP-1). The capability of compounds to affect the $\mathrm{NF}-\kappa \mathrm{B}$ pathway was also evaluated. We assessed the compounds-mediated regulation of cytokine and chemokine release by using Luminex X-MAP ${ }^{\circledR}$ technology in human primary peripheral blood mononuclear cells (PBMCs) upon LPS stimulation. We found that all compounds, also in the absence of electrophilic moieties, significantly suppressed the LPS-evoked secretion of pro-inflammatory cytokines such as TNF $\alpha$ and IL-1 $\beta$, but not of IL-8, in THP-1 cells. A reduction in the release of pro-inflammatory mediators similar to that induced by the compounds was also observed after siRNA mediated-Nrf2 knockdown, 
thus indicating that the attenuation of cytokine secretion cannot be directly ascribed to the activation of Nrf2 signaling pathway. Moreover, all compounds, with the exception of compound 1, attenuated the LPS-induced activation of the NF- $\mathrm{kB}$ pathway, by reducing the upstream phosphorylation of $1 \kappa B$, the NF- $\kappa B$ nuclear translocation, as well as the activation of NF-kB promoter. In human PBMCs, compound 4 and CURC attenuated TNF $\alpha$ release as observed in THP-1 cells, and all compounds acting as Nrf2 inducers significantly decreased the levels of MCP-1/CCL2, as well as the release of the proinflammatory cytokine IL-12. Altogether, the compounds induced a differential modulation of innate immune cytokine release, by differently regulating Nrf2 and NF- $\mathrm{B}$ intracellular signaling pathways.

Keywords: Nrf2, NF-кB, curcumin, antioxidant, inflammation, cytokine release, TNF $\alpha, \mathrm{MCP}-1$

\section{INTRODUCTION}

Nuclear factor (erythroid-derived 2)-like $2(\mathrm{Nrf2})$ is a transcription factor regulating the expression of about 250 genes encoding a network of cooperating enzymes involved in endobiotic and xenobiotic biotransformation reactions, antioxidant metabolism, protein degradation and regulation of inflammation (Hayes and Dinkova-Kostova, 2014). By governing such complex transcriptional networks, Nrf2 coordinates a multifaceted response to various forms of stress, maintaining a homeostatic intracellular environment. Several studies demonstrate that Nrf2 plays also a key role in the resolution of inflammatory processes. Consistently, Nrf2 is abundant in monocytes and granulocytes, proving its crucial involvement in immune response driven by these cell types. Data from the literature demonstrate that genetic or pharmacological activation of Nrf2 strongly suppresses the production of proinflammatory cytokines (Innamorato et al., 2008; Knatko et al., 2015; Kobayashi et al., 2016; Quinti et al., 2017) and Nrf2deficiency induces an exacerbation of inflammation in a variety of murine models such as sepsis, pleurisy, and emphysema (Iizuka et al., 2005; Ishii et al., 2005; Thimmulappa et al., 2006). However, while the contribution of Nrf2 in inflammatory processes has been widely recognized, the underlying molecular basis has not been completely clarified. Its anti-inflammatory activity has been related to several mechanisms, including crosstalk with the transcription factor nuclear factor- $\mathrm{\kappa B}$ (NF- $\mathrm{KB}$ ), the modulation of redox balance, and the direct down-regulation of some antioxidant response element (ARE)-dependent expression of pro-inflammatory cytokines, such as IL-6 and IL-1 13 (Kobayashi et al., 2016). Among them, the crosstalk between Nrf2 and NF-KB relies on both transcriptional and post-transcriptional mechanisms, allowing fine-tuning of dynamic responses to ever-changing environmental cues. NF- $\mathrm{KB}$ is a key transcription factor governing the expression of a plethora of genes involved in diverse biological processes, including immune and inflammatory responses, cell proliferation, death, angiogenesis, cell survival, and oncogenesis (Häcker and Karin, 2006; Perkins, 2007). In particular, NF-אB controls the transcription of genes encoding pro-inflammatory cytokines, such as TNF $\alpha$ and IL-1 $\beta$. In the absence of a stimulant, $\mathrm{NF}-\mathrm{KB}$ remains inactive and sequestrated in the cytoplasm by binding to an inhibitory protein, IкB. The exposure of cells to pro-inflammatory stimuli, such as cytokines and infectious agents, triggers the activation of the IKB kinase (IKK) complex that phosphorylates IKB protein on two serine residues. Phosphorylated I $\kappa \mathrm{B}$ is ubiquitinated and, subsequently, degraded via proteasome (Hayden et al., 2006; Perkins, 2007). The degradation of IкB allows $\mathrm{NF}-\mathrm{KB}$ translocation into the nucleus to drive the expression of target genes; within the nucleus it interacts with other transcription factors and transcriptional co-factors to regulate expression of an array of genes, many of which are involved in inflammatory signaling (e.g. cytokines, chemokines, adhesion molecules, and acute phase proteins) (Baeuerle and Baltimore, 1996). Notably, several pharmacological and genetic studies suggest a functional crosstalk between Nrf2 and NF- $\mathrm{KB}$ transcription factors, with a range of complex molecular interactions depending on the cell type and tissue context (Wardyn et al., 2015). A strong activity in both NF-KB and Nrf2 has been found fundamental for well-coordinated responses to counteract a cellular inflammatory status (Fusco et al., 2017; D’amico et al., 2019). Indeed, an imbalance between Nrf2 and NF- $\mathrm{BB}$ pathways has been associated with a variety of diseases ranging from neurodegeneration, cardiovascular and autoimmune disorders.

The transcriptional factor Nrf2, with its redox sensitive repressor Keap1 (Kelch-like ECH-associated protein 1), orchestrates adaptive responses to diverse forms of stress through regulatory cysteine switches. Thus, precise electrophilic addition is emerging as a valuable opportunity to shed light on previously untapped roles of this redox sensing system (Basagni et al., 2019). By combining into new chemical entities the hydroxycinnamoyl motif derived from curcumin and the allyl mercaptan moiety of garlic organosulfur compounds, we previously synthesized a set of molecules (compounds 1-3), carrying, with the exception of compound 3, a catechol moiety and/or an $\alpha, \beta$-unsaturated carbonyl group (Table 1). These (pro)electrophilic features were shown to be responsible for the activation of the Nrf2 pathway and the subsequent induction of ARE-dependent target genes, possibly by covalent conjugation with Keap1 cysteine sensors (Simoni et al., 2017; Serafini et al., 2019). Notably, alkylation of functionally significant cysteines of NF- $\mathrm{kB}$ was also shown to play a prominent role in the inhibition of pro-inflammatory transcriptional pathways (Kastrati et al., 2016), albeit alternative mechanisms have been proposed, such as the inhibition of IKK $\beta$, or 
TABLE 1 | Design strategy of electrophilic and non-electrophilic compounds.

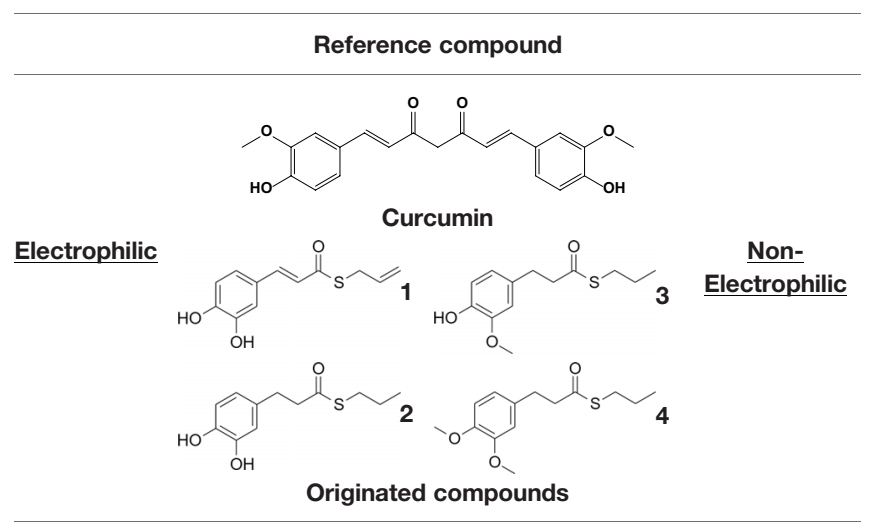

Compound 1: S-allyl (E)-3-(3,4-dihydroxyphenyl)prop-2-enethioate;

Compound 2: S-propyl 3-(3,4-dihydroxyphenyl)propanethioate;

Compound 3: S-propyl 3-(4-hydroxy-3-methoxyphenyl)propanethioate;

Compound 4: S-propyl 3-(3,4-dimethoxyphenyl)propanethioate.

promotion of RelA polyubiquitination and proteasomal degradation (Woodcock et al., 2018).

Herein, we considered the abovementioned compounds as valuable pharmacologic tools to explore the mechanistic connection between Nrf2 and NF- $\kappa$ B. To exclude possible oxidative activation of the methoxyphenol ring of compound 3 into reactive metabolites such as quinone methide, which could provide an additional electrophilic site (Luis et al., 2018), an additional new compound 4 was synthesized (Table 1).

In the present work, we investigated whether the modulation in Nrf2 pathway activation by our molecules was able to interfere with the LPS-induced secretion of pro-inflammatory cytokines, during immune stimulation, in a human immortalized monocytelike cell line (THP-1), a well-established cell model for the immune modulation approach (Chanput et al., 2014), using curcumin (CURC) as a reference compound. Moreover, the capability of compounds to affect the NF- $\kappa B$ intracellular pathway, a pivotal mediator of inflammatory responses and critical regulator of multiple aspects of both innate and adaptative immune functions, was also investigated. To validate the results obtained in THP-1 cells in a human primary cellular model, we assessed the regulation of cytokine and chemokine (e.g. IFN $\gamma$, IL-1 $\beta$, IL-4, IL-6, IL-8, IL-12 (p40), IL-12 (p70), IL-13, IL27, MCP-1, MCP-3, TNF $\alpha$ ) release by the described compounds, upon immune LPS stimulation, in human peripheral blood mononuclear cells (PBMCs), obtained from venous whole blood of healthy patients, by using Luminex X-MAP ${ }^{\circledR}$ technology.

Altogether, we demonstrated that compounds modulated the innate immune cytokine release, by differently regulating $\mathrm{Nrf} 2$ and NF- $\kappa$ B intracellular signaling pathways.

\section{MATERIALS AND METHODS}

\section{Compounds Synthesis}

Compounds 1-3 were synthesized according to procedures reported in (Simoni et al., 2016; Simoni et al., 2017); details on the newly synthesized compound 4 are reported here below.

\section{Synthesis of S-Propyl 3-(3,4-Dimethoxyphenyl) propanethioate (Compound 4)}

To a solution of compound $2(110 \mathrm{mg}, 0.46 \mathrm{mmol})$ in $1.80 \mathrm{~mL}$ of DMF potassium carbonate $(222.5 \mathrm{mg}, 1.61 \mathrm{mmol})$ and methyl iodide dropwise $(0.10 \mathrm{~mL}, 1.61 \mathrm{mmol})$ were added. The reaction mixture was left stirring at room temperature overnight. The reaction was quenched by adding $3 \mathrm{~mL}$ of water and the mixture obtained was further extracted with diethyl ether $(2 \times 5 \mathrm{~mL})$. Organic phases were collected, reunited, dried with anhydrous sodium sulphate and solvent was evaporated under vacuum. The crude oil was purified by column chromatography on silica gel using petroleum ether/ethyl acetate $(8 / 2)$ as mobile phase. 4 was obtained as colorless oil (110 mg, 89\%). 1H NMR (400 MHz, $\mathrm{CDCl} 3) \delta 6.74(\mathrm{~d}, \mathrm{~J}=8 \mathrm{~Hz}, 1 \mathrm{H}), 6.69-6.67(\mathrm{~m}, 2 \mathrm{H}), 3.82(\mathrm{~s}, 3 \mathrm{H})$, $3.80(\mathrm{~s}, 3 \mathrm{H}), 2.89(\mathrm{t}, \mathrm{J}=8 \mathrm{~Hz}, 2 \mathrm{H}), 2.83-2.77(\mathrm{~m}, 4 \mathrm{H}), 1.59-1.50$ $(\mathrm{m}, 2 \mathrm{H}), 0.91(\mathrm{t}, \mathrm{J}=8 \mathrm{~Hz}, 3 \mathrm{H}) .13 \mathrm{C} \mathrm{NMR}(100 \mathrm{MHz}, \mathrm{CDCl} 3) \delta$ $198.79,148.98,147.61,132.83,120.27,111.77,111.41,55.98$, $55.89,45.88,31.23,30.87,23.06,13.39$. MS [ESI+] m/z 291.10 $[\mathrm{M}+\mathrm{Na}]^{+}$.

Chromatographic separations were performed on silica gel columns (Kieselgel 40, 0.040-0.063 mm, Merck). Reactions were followed by TLC on Merck $(0.25 \mathrm{~mm})$ glass-packed precoated silica gel plates (60 F254), then visualized with a UV lamp. NMR spectra were recorded at $400 \mathrm{MHz}$ for $1 \mathrm{H}$ and $100 \mathrm{MHz}$ for $13 \mathrm{C}$ on a Varian VXR 400 spectrometer (Supplementary Figure 1). Chemical shifts $(\delta)$ are reported in parts per million (ppm) relative to tetramethylsilane (TMS), and spin multiplicities are given as s (singlet), br s (broad singlet), $d$ (doublet), $t$ (triplet), $q$ (quartet), or m (multiplet). Direct infusion ESI-MS mass spectra were recorded on a Waters ZQ 4000 and Xevo G2-XS QTof apparatus. Final compounds were $>95 \%$ pure as determined by High Performance Liquid Chromatography (HPLC) analyses. The analyses were performed under reversed-phase conditions on a Phenomenex Jupiter C18 $(150 \times 4.6 \mathrm{~mm}$ I.D. $)$ column, using a binary mixture of $\mathrm{H}_{2} \mathrm{O}$ /acetonitrile $(60 / 40$, v/v for $1 ; 50 / 50$, v/v for 2 and 3; 40/60, v/v for 4 ) as the mobile phase, UV detection at $\lambda=302 \mathrm{~nm}$ (for 1) or $254 \mathrm{~nm}$ (for 2-4), and a flow rate of $0.7 \mathrm{~mL} /$ min. Analyses were performed on a liquid chromatograph model PU-1587 UV equipped with a $20 \mu \mathrm{L}$ loop valve (Jasco Europe, Italy).

All compounds were solubilized in DMSO (dimethyl sulfoxide) at stock concentrations of $10 \mathrm{mM}$, frozen $\left(-20^{\circ} \mathrm{C}\right)$ in aliquots and diluted in culture medium immediately prior to use. For each experimental setting, a stock aliquot was thawed and diluted to minimize repeated freeze and thaw damage. The final concentration of DMSO in culture medium was less than $0.1 \%$.

\section{Reagents}

CURC (\#08511) was $\geq 98 \%$ pure (HPLC) and purchased by Sigma-Aldrich (Merck KGaA, Darmstadt, Germany). Cell culture media and all supplements were purchased from Sigma Aldrich (Merck KGaA, Darmstadt, Germany). Rabbit polyclonal anti-human Nrf2 (NBP1-32822) and anti-human HO-1 (NBP131341) antibodies were purchased from Novus (Biotechne, Minneapolis USA). Mouse monoclonal anti-human $\operatorname{I\kappa B} \alpha$ $(\# 4814 \mathrm{~T})$, mouse monoclonal anti-human phospho-IкB $\alpha$ 
(Ser32/36) (\#9246T), and rabbit monoclonal anti-human NF-кB p65 (D14E12) XP ${ }^{\circledR}$ (\#8242) were purchased from Cell Signaling (Cell Signaling Technology, Danvers, MA, USA). Mouse monoclonal anti-human lamin A/C (612162) antibody was purchased from BD Biosciences (Franklin Lakes, NJ, USA). Mouse anti-human $\alpha$-tubulin (sc-5286) was purchased from Sigma-Aldrich (Merck KGaA, Darmstadt, Germany). Peroxidase conjugate-goat anti-mouse (A4416) was purchased from SigmaAldrich (Merck KGaA, Darmstadt, Germany). Anti-rabbit peroxidase-linked antibody (\#7074) was purchased from Cell Signaling (Cell Signaling Technology, Danvers, MA, USA). Lipopolysaccharide (LPS) from Escherichia coli O111:B4 (L2630) was purchased from Sigma-Aldrich (Merck KGaA, Darmstadt, Germany). The proteasome inhibitor MG132 (474790) was purchased from Calbiochem (San Diego, CA).

\section{Cell Culture and Treatments}

Human THP-1 cells were purchased from the European Collection of Authenticated Cell Cultures (ECACC, Salisbury, UK) and diluted to $10^{6}$ cells/mL in RPMI 1640 medium supplemented with $10 \%$ heat-inactivated Fetal Bovine Serum (FBS), $2 \mathrm{mM}$ glutamine, $0.1 \mathrm{mg} / \mathrm{mL}$ streptomycin, $100 \mathrm{IU} \cdot \mathrm{mL}$ penicillin, and $0.05 \mathrm{mM} 2$-mercaptoethanol (complete medium) and maintained at $37^{\circ} \mathrm{C}$ in $5 \% \mathrm{CO}_{2}$-containing and $95 \%$ air atmosphere. The experiments were carried out on passages 5-15. Cells were treated as reported in figure legends. Control cells were exposed only to solvent (DMSO).

\section{Cell Viability}

The mitochondrial dehydrogenase activity that reduces 3-(4,5dimethylthiazol-2-yl)-2,5-diphenyl-tetrazolium bromide (MTT, Sigma Aldrich, Merck KGaA, Darmstadt, Germany) was used to determine cell viability using a quantitative colorimetric assay (Kumar et al., 2018). At day 0, THP-1 cells were plated in 96-well plates at a density of $50 \times 10^{3}$ viable cells per well. After treatment, according to the experimental setting, cells were exposed to an MTT solution (1 $\mathrm{mg} / \mathrm{mL}$ ) in complete medium. After $4 \mathrm{~h}$ of incubation with MTT, cells were lysed with sodium dodecyl sulfate (SDS) for $24 \mathrm{~h}$ and cell viability was quantified by reading absorbance at $570 \mathrm{~nm}$ wavelength, using Synergy HT multi-detection microplate reader (Bio-Tek, Winooski, VT, USA).

\section{Subcellular Fractionation for Nrf2 and NF- $\kappa B$ Nuclear Translocation}

The expression of Nrf2 and NF- $\mathrm{KB}$ in nuclear THP-1 lysates was assessed by Western blot analysis. Suspended cells were collected, centrifugated, and washed twice with ice-cold PBS (phosphate buffered saline), and, subsequently, homogenized 20 times using a glass-glass homogenizer in ice-cold fractionation buffer (20 $\mathrm{mM}$ Tris/HCl pH 7.4, 2 mM EDTA, $0.5 \mathrm{mM}$ EGTA, $0.32 \mathrm{M}$ sucrose, $50 \mathrm{mM} \beta$-mercaptoethanol). The homogenate was centrifuged at $300 \times g$ for $5 \mathrm{~min}$ to obtain the nuclear fraction. An aliquot of the nuclear extract was used for protein quantification by the Bradford method, whereas the remaining sample was boiled at $95^{\circ} \mathrm{C}$ for 5 min after dilution with $2 \mathrm{X}$ sample buffer (125 mM Tris- $\mathrm{HCl}$ pH 6.8, 4\% SDS, 20\% glycerol, $6 \% \beta$-mercaptoethanol, $0.1 \%$ bromophenol blue). Equivalent amounts of nuclear extracted proteins $(30 \mu \mathrm{g})$ were subjected to polyacrylamide gel electrophoresis and immunoblotting, as described below.

\section{Immunodetection of Nrf2, HO-1, p-IкB $\alpha$, I $\mathrm{kB} \alpha$, and NF- $\mathrm{kB}$}

The expression of Nrf2, HO-1, p-I $\mathrm{HB} \alpha$, I $\kappa \mathrm{B} \alpha$, and NF- $\kappa \mathrm{B}$ in whole cell lysates or nuclear extracts was assessed by Western blot analysis. After treatments, suspended cells were collected, centrifugated, and washed twice with ice-cold PBS, lysed by the addition of ice-cold homogenization buffer $(50 \mathrm{mM}$ Tris- $\mathrm{HCl} \mathrm{pH}$ 7.5, $150 \mathrm{mM} \mathrm{NaCl}, 5 \mathrm{mM}$ EDTA, 0.5\% Triton X-100 and proteasephosphatase inhibitors mix). Samples were sonicated and centrifuged at $13,000 \times g$ for $10 \mathrm{~s}$ at $4^{\circ} \mathrm{C}$. The resulting supernatants were transferred into new tubes, and protein content was determined by Bradford method. After that, the samples were boiled at $95^{\circ} \mathrm{C}$ for $5 \mathrm{~min}$ after dilution with $5 \mathrm{X}$ sample buffer. For Western blot analysis, equivalent amounts of both total and nuclear extracts $(30 \mu \mathrm{g})$ were electrophoresed in $10 \%$ acrylamide gel, under reducing conditions, then, electroblotted into PVDF membranes (Sigma Aldrich, Merck KGaA, Darmstadt, Germany), blocked for $1 \mathrm{~h}$ with $5 \% \mathrm{w} / \mathrm{v}$ bovine serum albumin (BSA) in TBS-T $(0.1 \mathrm{M}$ Tris- $\mathrm{HCl} \mathrm{pH}$ $7.4,0.15 \mathrm{M} \mathrm{NaCl}$, and $0.1 \%$ Tween 20 ), and incubated overnight at $4^{\circ} \mathrm{C}$ with primary antibodies diluted in $5 \% \mathrm{w} / \mathrm{v}$ BSA in TBS-T. The proteins were visualized using primary antibodies for $\mathrm{Nrf} 2$

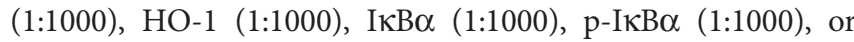
NF- $\kappa B$ (1:1000). Detection was carried out by incubation with secondary horseradish peroxidase-conjugated antibodies (1:5000) diluted in 5\% w/v BSA in TBS-T for $1 \mathrm{~h}$ at room temperature. Membranes were subsequently washed three times with TBS-T and proteins of interest were visualized using an enhanced chemiluminescent reagent (Pierce, Rockford, IL, USA). $\alpha$ tubulin and lamin $\mathrm{A} / \mathrm{C}$ were performed as controls for gel loading.

\section{Small Interference RNA (siRNA) for Nrf2}

Nrf2 siRNA designed for the human gene Nrf2 was purchased from Sigma Aldrich, Merck KGaA (Darmstadt, Germany). A scrambled siRNA, without known homology with any gene, was used as negative control (Sigma Aldrich, Merck KGaA, Darmstadt, Germany). RNA interference experiments in THP1 cells were performed by transient transfection for $24 \mathrm{~h}$, using RNAiMAX Lipofectamine (Invitrogen, Thermo Fisher Scientific, Waltham, MA, USA), according to manufacturer's protocol. To confirm Nrf2 silencing, the proteasome inhibitor MG132 (Calbiochem) was added to the medium of selected plates at a final concentration of $5 \mu \mathrm{M}$. After $24 \mathrm{~h}$, cells were analyzed for Nrf2 expression by Western blot analysis.

\section{Enzyme-Linked Immunosorbent Assay (ELISA) Determination of TNF $\alpha$, IL-8, and IL-1 $\beta$}

THP-1 cells were treated with compounds 1-4 and CURC at a concentration of $5 \mu \mathrm{M}$ for $24 \mathrm{~h}$, and then stimulated with LPS for $3 \mathrm{~h}$, as described in the legends to figures. TNF $\alpha$, IL- 8 , and IL- $1 \beta$ released from THP-1 cells were measured in cell-free supernatants 
obtained by centrifugation at $250 \mathrm{x} \mathrm{g}$ for $5 \mathrm{~min}$ and immediately processed for ELISA, according to the manufacturer's protocol. TNF $\alpha$, IL- 8 and IL-1 $\beta$ production was assessed by specific sandwich ELISA (Invitrogen, Thermo Fisher Scientific, Waltham, MA, USA; Immunotools GmbH, Friesoythe, Germany). Results were expressed as stimulation index. The limit of detection under optimal conditions was $4 \mathrm{pg} / \mathrm{mL}$ for TNF $\alpha, 2.6 \mathrm{pg} / \mathrm{mL}$ for IL-8, and $18 \mathrm{pg} / \mathrm{mL}$ for IL-1ß.

\section{Plasmid DNA Preparation, Transient Transfections, and Luciferase Assay}

Plasmids for transfections were purified with the HiSpeed ${ }^{\circledR}$ Plasmid Midi Kit (Qiagen, Valencia, CA). DNA was quantified and assayed for purity using QuantusTM Fluorometer (Promega, Madison, WI). Transient transfections were performed in 12multiwell culture plates; for each well $5 \times 10^{5}$ cells were seeded in RPMI 1640 complete medium. Transfections were carried out using Lipofectamin 2000 Transfection Reagent (Invitrogen, Thermo Fisher Scientific, Waltham, MA, USA), according to manufacturer's instructions. pGL4.32 vector (E8491, Promega, Madison, WI) luciferase-reporter construct plasmid DNA was co-transfected with pRL-TK Renilla (E2241, Promega, Madison, WI) luciferase expressing vector to measure transfection efficiency, as described in Buoso et al. (2019). During transfection THP-1 cells were incubated at $37^{\circ} \mathrm{C}$ in $5 \% \mathrm{CO}_{2}$ overnight and, then, treated with $5 \mu \mathrm{M}$ compounds and CURC for $24 \mathrm{~h}$ and, then, stimulated with $10 \mathrm{ng} / \mathrm{mL}$ LPS for $6 \mathrm{~h}$. At the end of the treatments, cells were lysed with Passive Lysis Buffer provided by Dual-Luciferase ${ }^{\circledR}$ Reporter Assay System, following manufacturer's instructions (Promega, Madison, WI). The luminescent signals were measured using a 20/20 Luminometer with $10 \mathrm{~s}$ of integration (Turner BioSystems, Sunnyvale, CA).

\section{PBMCs Purification and Culture}

Human peripheral blood mononuclear cells (PBMCs) were obtained from the blood of five (5) healthy individuals (mean age \pm SD: $71 \pm 5.22$ years; gender: 3 females and 2 males) satisfying the SENIEUR standard protocol for immuno-gerontological studies (Pawelec et al., 2001). Subjects having a history or physical signs of atherosclerosis or inflammation were excluded. All subjects gave written informed consent in accordance with the Declaration of Helsinki (Ethical Committee Project approval: Fulop_2019-2877). Heparinized blood was subjected to density gradient centrifugation over Ficoll-Paque Plus medium (GE Healthcare Life Sciences, Marlborough, MA, USA) as described in (Le Page et al., 2017). Briefly, PBS-diluted blood was carefully layered onto the FicollPaque density gradient and centrifuged for $20 \mathrm{~min}$ at $400 \mathrm{x} g$ at slow acceleration and with the brake off at room temperature. After centrifugation, the PBMCs layer, consisting of monocytes, $\mathrm{T}$ and $\mathrm{B}$ lymphocytes, was collected and washed three times with fresh PBS. Cell viability, assessed by Trypan blue exclusion, was more than 95\%. For experiments, PBMCs were resuspended at a density of $1 \times 10^{6}$ cells $/ \mathrm{mL}$ in complete culture medium consisting of RPMI 1640 supplemented with $10 \%$ heat-inactivated FBS, $2 \mathrm{mM}$ glutamine, $0.1 \mathrm{mg} / \mathrm{mL}$ streptomycin and $100 \mathrm{IU} \mathrm{mL}$ penicillin and maintained at $37^{\circ} \mathrm{C}$ in $5 \% \mathrm{CO}_{2}$ and $95 \%$ air atmosphere.

\section{Luminex X-MAP ${ }^{\circledR}$ Assay}

Human cytokine MILLIPLEX ${ }^{\circledR}$ MAP Kit (customized for IFN $\gamma$, IL-1 $\beta$, IL-4, IL-6, IL-8, IL-12 (p40), IL-12 (p70), IL-13, IL-27, MCP-1, MCP-3, TNF $\alpha$ ) was purchased from Millipore-Sigma (Merck KGaA). The assay was performed in a 96-well plate and all reagents were prepared according to the manufacturer's instructions. Each well was cleaned and pre-wet with $200 \mu \mathrm{L}$ of wash buffer on plate at $450 \mathrm{rpm}$ during $10 \mathrm{~min}$ at RT. Wash buffer was removed by inverting the plate. Assay buffer, matrix solution or culture medium was used as a blank, each standard from a range of concentrations (different for each analyte), quality controls and samples were added to the appropriate wells. The mixed magnetic microbead solution was sonicated and vortexed prior to adding $25 \mu \mathrm{L}$ into each well. The plates were sealed and incubated with agitation on a plate shaker at 750 rpm overnight at $4^{\circ} \mathrm{C}$ in a darkroom. Plates were put on the magnetic support to retain microbeads, then fluid was removed by inverting the plate to avoid touching the beads. Each well was washed three times with $200 \mu \mathrm{L}$ of wash buffer with a plate shaker at $450 \mathrm{rpm}$ for $30 \mathrm{~s}$ at RT. $25 \mu \mathrm{L}$ of biotinylated detection antibodies were added per well, and plates were incubated in dark room at RT on a plate shaker at $750 \mathrm{rpm}$ for $1 \mathrm{~h}$. Then, $25 \mu \mathrm{L}$ of streptavidin-phycoerythrin solution were added to each well, and plates were incubated on a plate shaker at $750 \mathrm{rpm}$ for $30 \mathrm{~min}$ at RT and protected from light. Plates were washed three times with $200 \mu \mathrm{L}$ of wash buffer. Microbeads were resuspended in $150 \mu \mathrm{L} /$ well of sheath fluid on a plate shaker at $450 \mathrm{rpm}$ for 5 min at RT. Data were acquired on a Luminex ${ }^{\circledR}$ 200TM System using the Luminex xPonent ${ }^{\circledR}$ software. An acquisition gate of between 8,000 and 15,000 was set to discriminate against any doublet events and ensure that only single microbeads were measured. Fifty beads/assay were collected and median fluorescence intensities (MFIs) were measured. Sensitivity limits (in $\mathrm{pg} / \mathrm{mL}$ ) were 0.86 for IFN $\gamma ; 0.52$ for IL-1 $\beta ; 0.2$ for IL-4; 0.14 for IL-6; 0.52 for IL-8; 2.24 for IL-12 (p40); 0.88 for IL12 (p70); 2.58 for IL-13; 50.78 for IL-27; 3.05 for MCP-1; 8.61 for MCP-3 and 5.39 for TNF $\alpha$. MFIs were converted to concentrations using the equation of standard range of the appropriate cytokine using Milliplex ${ }^{\circledR}$ Analyst 5.1 Software.

\section{Densitometry and Statistics}

All the experiments were performed at least three times with representative results being shown. Data are expressed as mean \pm SEM. The relative densities of the acquired images of Western blotting bands were analyzed with ImageJ software. Statistical analyses were performed using Prism software (GraphPad software, San Diego, CA, USA; version 8.0). Statistical differences were determined by analysis of variance (ANOVA) followed, when significant, by an appropriate post hoc test, as indicated in the figure legends. In all reported statistical analyses, effects were designated as non-significant for $\mathrm{p}>0.05$, significant $\left.{ }^{*}\right)$ for $p<0.05$ or less as indicated.

\section{Quantum Mechanics Calculations}

The study for the conformational freedom of compounds 1-4 was conducted with the software Gaussian 09 (Gaussian Inc., 
Wallingford, CT, USA; Revision A.02). Each molecule underwent a protocol of geometrical optimization, involving an increasing level of precision of basis sets [i.e., from 3-21 (Binkley et al., 1980) to $6-31 G^{*}$ (Petersson and Al-Laham, 1991)], with the HartreeFock (HF) method (Kohn and Sham, 1965). The "Scan" functionality was used to estimate the barrier hindering conformational variability in the compounds for two different dihedral angles (Supplementary Figure 2). During this step, a Møller-Plesset correlation energy correction truncated at the second order (MP2) (Møller and Plesset, 1934) was added to the HF method and the torsions were rotated by intervals of 5 degrees until they completed the 360 degree turn. For each of these steps, the dihedral angle under study was fixed and the energy of the structure was computed after few steps of minimization.

\section{RESULTS}

\section{Cellular Toxicity of Compounds}

The cytotoxicity of compounds 1-4 was assessed by MTT assay in THP-1 cells, in comparison with CURC, as a reference compound. Cells were exposed to compounds 1-4 and CURC at concentrations of $1 \mu \mathrm{M}, 2.5 \mu \mathrm{M}, 5 \mu \mathrm{M}$, and $10 \mu \mathrm{M}$ for $24 h$. Consistently with our previous data on a different cellular model (Simoni et al., 2016; Simoni et al., 2017; Serafini et al., 2019; Catanzaro et al., 2020), all the compounds were well-tolerated, with a slight reduction of cell viability of about $10 \%$ observed for compounds 3 and 4 (Figure 1). Based on these results and according to our previous investigations (Simoni et al., 2017; Serafini et al., 2019; Catanzaro et al., 2020), all further experiments were conducted using the concentration of $5 \mu \mathrm{M}$.

\section{Modulation of Nrf2 Nuclear Translocation and HO-1 Target by Compounds}

$\mathrm{Nrf} 2$ is a redox-sensitive transcription factor orchestrating the expression and coordinated induction of a wide battery of genes encoding phase II and detoxifying enzymes. Under unstressed conditions, Nrf2 is retained in the cytoplasm by its negative repressor Keap1 and rapidly subjected to ubiquitination and proteasomal degradation, mediated by the binding of Keap 1 to the Cul3/Rbx1 E1 ubiquitin ligase complex (Niture et al., 2014). After exposure to oxidative and/or electrophilic stimuli, Nrf2 is released from structurally modified Keap1 and translocates into the nucleus, forms a heterodimer with one of the small musculoaponeurotic fibrosarcoma (Maf) proteins, and activates the ARE-mediated expression of cytoprotective genes. Since Nrf2 nuclear translocation is a fundamental step for the complete activation of its pathway, we tested the ability of compounds to induce the nuclear translocation of Nrf2 in THP-1 cells, by comparing their effects to CURC, used as a positive control.

Notably, evidence from the literature demonstrates that proelectrophilic (catechol) and/or electrophilic moieties (the Michael acceptor $\alpha, \beta$-unsaturated carbonyl group) are important structural functions necessary for the induction of the Nrf2 pathway (Tanigawa et al., 2007; Satoh et al., 2013). Compounds were synthesized and screened to identify the structural moieties

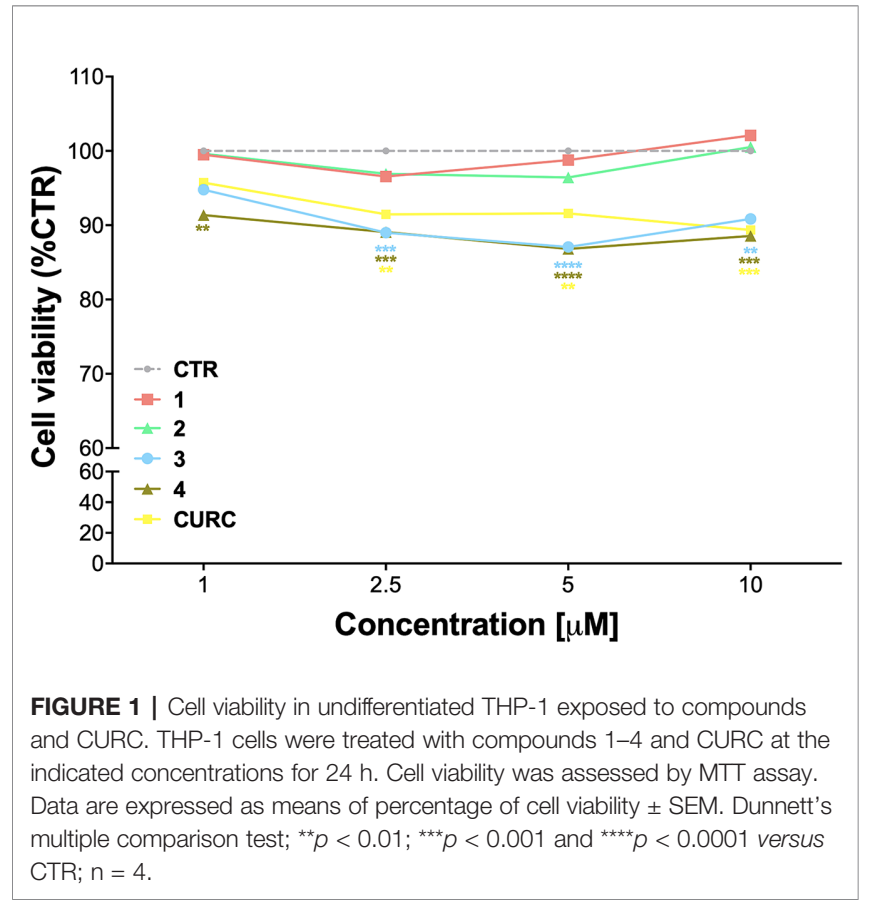

responsible for the activation of $\mathrm{Nrf} 2$ and its downstream signaling pathway. The four compounds investigated in this study differ from each other by the presence or absence of the mentioned key functional groups (as shown in Table 1). Indeed, while compound 1 provides the catechol moiety, as well as the Michael acceptor group, 2 displays only the catechol moiety. Conversely, compounds 3 and 4 lack for both the Michael acceptor group and the catechol function.

Thus, THP-1 cells were treated with DMSO as vehicle control, compounds 1-4 and CURC at a concentration of $5 \mu \mathrm{M}$ for $3 h$. After treatment, Nrf2 nuclear content was assessed by Western blot analysis. As shown in Figure 2A, compounds 1, 2, as well as CURC, significantly induced Nrf2 nuclear translocation, whereas compounds 3 and 4 did not increase Nrf2 nuclear content (Figure 2A). Such results are consistent with our previous work (Simoni et al., 2017; Serafini et al., 2019; Catanzaro et al., 2020), where the ability of compounds 1 and 2, but not 3, to activate the Nrf2 pathway in SH-SY5Y and ARPE-19 cells suggested that the addition of Keap1 nucleophilic cysteines to (pro)electrophilic portions of the molecule could represent the initiating event. The finding that the newly synthesized molecule, compound 4, was also unable to induce Nrf2 nuclear translocation corroborates this hypothesis.

To demonstrate the downstream activation of the Nrf2 signaling pathway, the protein amount of HO-1, one of the main targets of Nrf2, was evaluated by Western blot analysis. THP-1 cells were treated with DMSO as vehicle control, compounds 1-4 and CURC at a concentration of $5 \mu \mathrm{M}$ for $24 h$. As shown in Figure 2B, compounds 1, 2 and CURC positively modulated HO-1 protein levels, confirming the activation of the Nrf2 pathway. In contrast, compounds 3 and 4 did not affect the protein amount of HO-1 in THP-1 whole cell lysates, confirming their inability to promote Nfr2 pathway activation. 


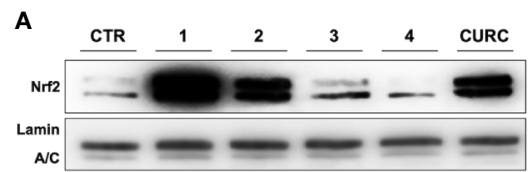

B
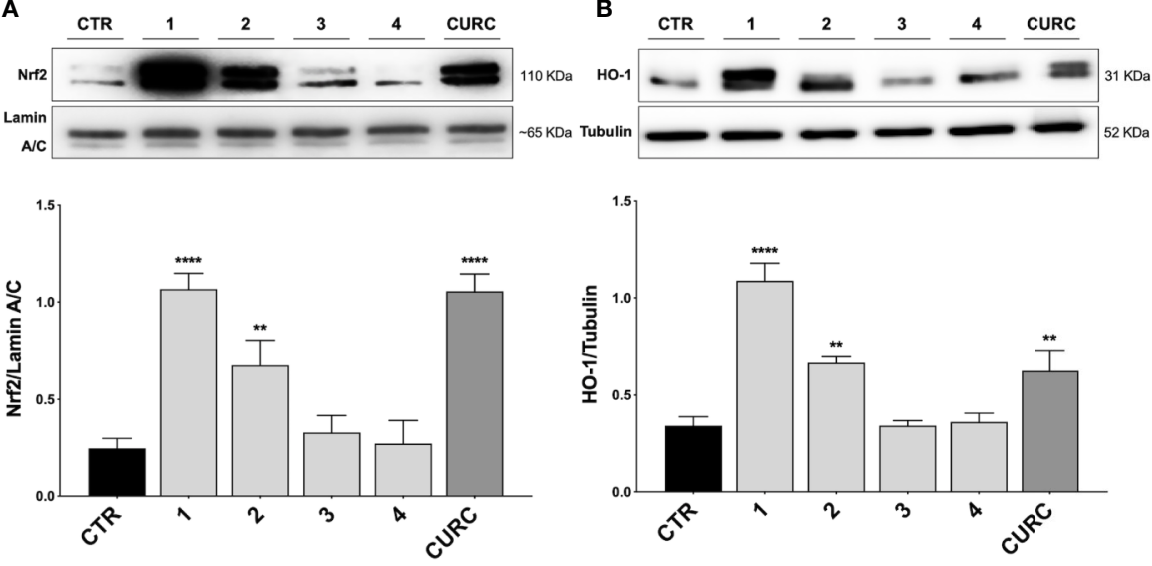

FIGURE 2 | Nrf2 nuclear translocation and modulation of HO-1 protein content in THP-1 cells. (A) THP-1 cells were treated with compounds 1-4 and CURC at a concentration of $5 \mu \mathrm{M}$ for $3 \mathrm{~h}$. After isolation, nuclear extracts were examined by Western blot analysis and Nrf2 expression was determined using an anti-Nrf2 antibody. Anti-lamin A/C was used as protein loading control. Results are shown as means of Nrf2/lamin A/C ratio \pm SEM. Dunnett's multiple comparison test; ${ }^{* *} p<0.01$ and ${ }^{* \star * *} p<0.0001$ versus CTR; $n=5-7$. (B) Total protein extracts of THP-1 cells, treated with compounds $1-4$ and CURC at the concentration of $5 \mu M$ for $24 \mathrm{~h}$, were analyzed for HO-1 protein content by Western blot analysis. Anti-tubulin was used as protein loading control. Results are shown as means of HO-1/ Tubulin ratio \pm SEM. Dunnett's multiple comparison test; ${ }^{\star \star} p<0.01$ and ${ }^{* \star \star *} p<0.0001$ versus CTR; $\mathrm{n}=7$.

\section{Compounds Attenuate TNF $\alpha$ and IL-1 $\beta$, but Not IL-8 Release, in LPS-Stimulated THP-1 Cells}

To investigate the immunomodulatory potential of compounds acting as Nrf2 inducers, we exposed THP-1 cells to LPS from E. coli, resulting in enhanced production and secretion of proinflammatory mediators (Supplementary Figure 3 and Figure 4A). Thus, THP-1 cells were treated with DMSO as vehicle control, compounds $1-4$ and CURC at a concentration of $5 \mu \mathrm{M}$ for $24 h$ and, then, exposed to $10 \mathrm{ng} / \mathrm{mL}$ LPS for $3 h$ in order to evoke the inflammatory response (Figure 3). TNF $\alpha$ (Figure 3A) and IL-8 protein release (Figure 3B ) were measured by ELISA in the supernatants of LPS-stimulated THP-1 cells. Notably, all compounds, independently from their ability to act as Nrf2 inducers, significantly reduced $\mathrm{TNF} \alpha$ protein release into cell culture medium (Figure 3A). In contrast, in the same experimental setting, all compounds, as well as CURC, did not affect IL-8 protein release into cell culture medium (Figure 3B).

We further investigated the effects of compounds 1-4 and CURC on the release of the pro-inflammatory mediator IL- $1 \beta$ upon stimulation. Unlike TNF $\alpha$ and IL-8, no increase in IL- $1 \beta$ protein release was observed in THP-1 cells exposed to $10 \mathrm{ng} / \mathrm{mL}$ LPS, but only after stimulation with $1 \mu \mathrm{g} / \mathrm{mL}$ LPS for $3 h$, as reported in Figure 4A. Then, THP-1 cells were treated with DMSO as vehicle control, compounds 1-4 and CURC at a concentration of $5 \mu \mathrm{M}$ for $24 h$, exposed to $1 \mu \mathrm{g} / \mathrm{mL}$ LPS for $3 h$ to promote the inflammatory response, and tested for IL-1 $\beta$ release by ELISA (Figure 4B). All the compounds, as well as
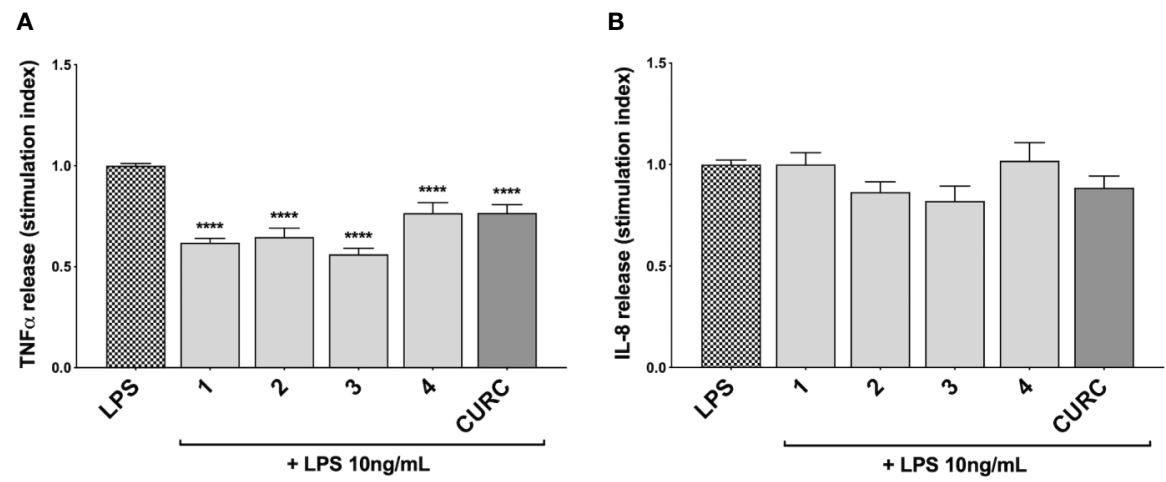

FIGURE 3 | Modulation of TNF $\alpha$ and IL-8 release in LPS-stimulated THP-1 cells exposed to compounds. THP-1 cells were treated with compounds 1-4 and CURC at a concentration of $5 \mu \mathrm{M}$ for $24 \mathrm{~h}$, and then stimulated with $10 \mathrm{ng} / \mathrm{mL}$ LPS for $3 \mathrm{~h}$. TNF $\alpha$ (A) and IL-8 (B) protein release was measured in THP-1 supernatants by ELISA. Data are presented as means of stimulation index \pm SEM. Dunnett's multiple comparison test; ${ }^{\star \star \star \star} p<0.0001$ versus CTR; $n=5$. 
CURC, significantly reduced IL- $1 \beta$ protein release into cell culture medium. As observed for $\mathrm{TNF} \alpha$, both compounds acting as Nrf2 inducers (1 and 2) and those inactive on the Nrf2 pathway (3 and 4) counteracted the LPS-driven inflammatory response, thus suggesting the involvement of different intracellular pathways.

\section{siRNA Mediated Nfr2 Knockdown Does Not Affect TNF $\alpha$ Release in LPS- Stimulated THP-1 Cells}

Based on the effect elicited by compounds 3 and 4 on cytokine release, the Nrf2 gene was knocked down by siRNA in THP-1 cells with the aim to evaluate the weight of the Nrf2 pathway in proinflammatory cytokine modulation upon LPS stimulation. Accordingly, cells were transfected with scrambled (siRNA ${ }_{\text {CTR }}$ ) and Nrf2 siRNA ( siRNA $_{\mathrm{Nrf} 2}$ ) for $24 h$ and the proteasome inhibitor MG132 $(5 \mu \mathrm{M})$ was added $4 h$ before the end of the experiment to the medium of selected plates in order to assess Nrf2 silencing. Nrf2 shows a short half-life, with a rapid ubiquitin-proteasomemediated degradation (Kobayashi and Yamamoto, 2006). Thus, to properly appreciate Nrf2 silencing, we blocked Nrf2 degradation using the proteasome inhibitor MG132. After treatments, the Nrf2 protein content was measured in whole cell lysates by Western blot analysis. As reported in Figure $\mathbf{5 A}$, the proteasome inhibitor MG132 induced an increase in Nrf2 protein levels in comparison with control. No statistically significant difference in Nrf2 protein levels between wild type (WT) and scrambled treated cells, treated with MG132, was found, whereas a marked decrease in Nrf2 protein content between $\mathrm{WT}$ and siRNA $\mathrm{Nrf}_{2}$-treated cells was observed (Figure 5A). Then, WT and siRNA $A_{\mathrm{Nrf2}}$ cells were treated for $24 h$ with $5 \mu \mathrm{M}$ of selected compounds (the Nrf2 inducer 1 , and the inactive 3 and 4), stimulated with $10 \mathrm{ng} / \mathrm{mL}$ LPS for $3 h$ to evoke the inflammatory response, and analysed for TNF $\alpha$ release by ELISA. Notably, all the selected compounds significantly suppressed LPS-induced release of TNF $\alpha$ both in WT and siRNA ${ }_{\mathrm{Nrf2}}$ cells (Figure $5 \mathbf{B}$ ), indicating that the observed reduction in pro-inflammatory cytokines release upon
LPS stimulation, cannot be explained on the basis of the activation of Nrf2 pathway.

\section{Modulation of the NF-кB Cellular Pathway by Compounds}

To better understand the mechanism of action underlying the reduction of cytokines induced by compounds, we investigated their potential effect on the NF- $\kappa \mathrm{B}$ pathway. Exposure of THP-1 cells to LPS from $E$. coli resulted in activation of the NF- $\mathrm{BB}$ transcription factor (Gomes et al., 2015; Sakai et al., 2017). To assess the effect of compounds on the NF- $\mathrm{KB}$ signaling pathway, we investigated the modulation of the upstream signaling molecule I $\mathrm{B} \mathrm{B} \alpha$. In our experimental setting, THP-1 cells were treated with DMSO as vehicle control, compounds 1-4 and CURC at a concentration of $5 \mu \mathrm{M}$ and, then, stimulated for $45 \mathrm{~min}$ with $10 \mathrm{ng} / \mathrm{mL}$ LPS. The phosphorylation of $\mathrm{I} \kappa \mathrm{B} \alpha$ was measured in whole cell lysates by Western blot analysis. As shown in Figure 6A, LPS stimulation markedly increased the level of $\mathrm{p}-\mathrm{I} \kappa \mathrm{B} \alpha$ compared to controls, whereas treatments with compounds 2, 3, 4, and CURC significantly prevented $\mathrm{I} \kappa \mathrm{B} \alpha$ phosphorylation, thus indicating that they might hinder the activation of the NF- $\kappa \mathrm{B}$ pathway by preventing I $\mathrm{I} \mathrm{B} \alpha$ phosphorylation. Compound 1 did not produce statistically significant results in our experimental setting, although a slight trend to decrease in I $\mathrm{B} \alpha$ phosphorylation could be observed (Figure 6A).

To further evaluate the capability of compounds to influence NF- $\kappa B$ nuclear translocation, THP-1 cells were treated with vehicle, $5 \mu \mathrm{M}$ compounds $1-4$ and CURC and, then, stimulated for $1 \mathrm{~h}$ and $30 \mathrm{~min}$ with $10 \mathrm{ng} / \mathrm{mL}$ LPS, as inflammatory stimulus promoting NF- $\mathrm{\kappa B}$ nuclear translocation. Compounds 3 and 4, and CURC markedly suppressed NF- $\kappa \mathrm{B}$ nuclear translocation, whereas compound 2 acted to a lower extent (Figure 6B). Compound 1 did not produce statistically significant results in our experimental setting, although a slight trend to decrease could be observed (Figure 6B).
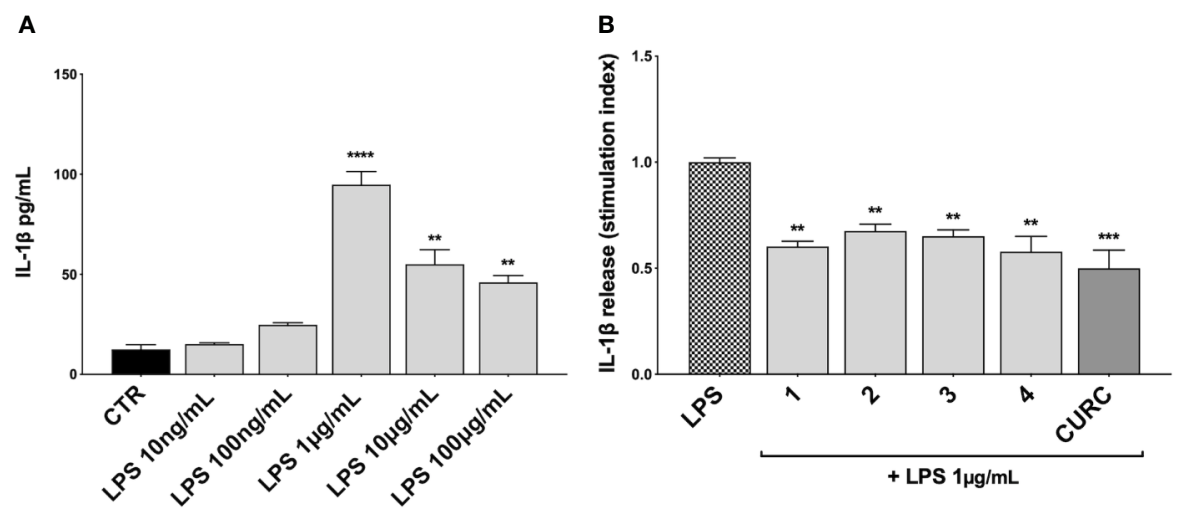

FIGURE 4 | Modulation of IL-1 $\beta$ release in LPS-stimulated THP-1 cells. (A) IL-1 $\beta$ protein secretion was measured in THP-1 cell supernatants stimulated with LPS at the indicated concentrations for $3 \mathrm{~h}$. At the end of the treatment, IL-1 $\beta$ protein release was assessed by ELISA. Data are presented as means of released picograms per $\mathrm{mL}(\mathrm{pg} / \mathrm{mL}) \pm \mathrm{SEM}$. Dunnett's multiple comparison test; ${ }^{* \star} p<0.01$ and ${ }^{\star \star \star *} p<0.0001$ versus $\mathrm{CTR} ; \mathrm{n}=3$. (B) IL-1 $\beta$ protein release was measured in THP-1 cells supernatants, treated for $24 \mathrm{~h}$ with compounds $1-4$ and CURC at a concentration of $5 \mu \mathrm{M}$ and then stimulated with $1 \mu \mathrm{g} / \mathrm{mL}$ LPS for $3 \mathrm{~h}$. The level of IL-1 $\beta$ was assessed by ELISA. Data are presented as means of stimulation index \pm SEM. Dunnett's multiple comparison test; ${ }^{* \star} p<0.01$ and ${ }^{* \star *} p<0.001$ versus CTR; $n=3$. 

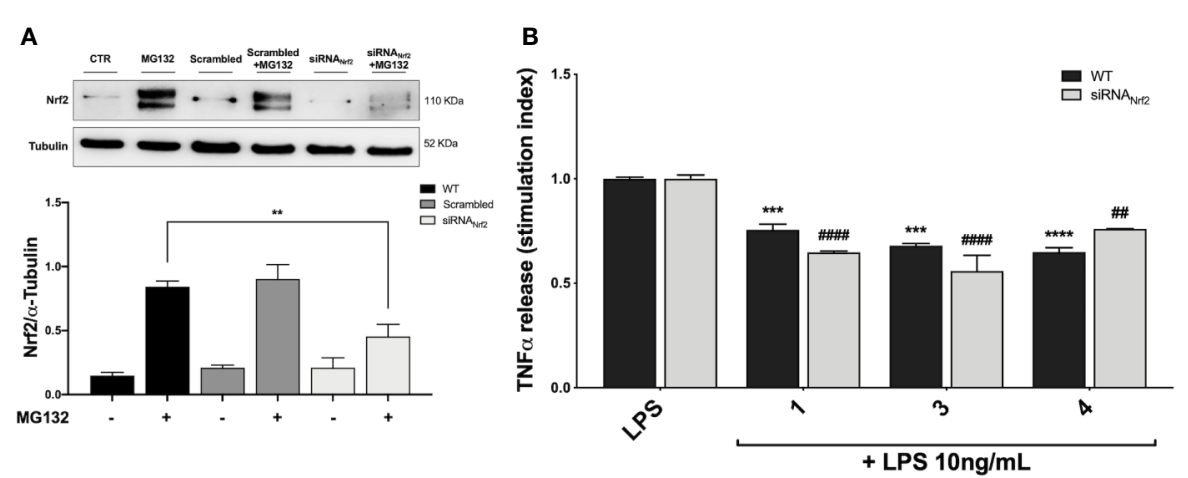

FIGURE 5 | Optimization of Nfr2-silenced THP-1 model (A) and effect of Nrf2-knockdown on modulation of TNF $\alpha$ release by compounds 1, 3 and 4, upon LPS stimulation (B). (A) THP-1 cells were treated either with vehicle (WT), scrambled or siRNA $\mathrm{Nr}_{2}$ for $24 \mathrm{~h}$. Where indicated MG132 was added $4 \mathrm{~h}$ before the end of the experiment to block the proteasomal degradation of Nrf2. After treatments, Nrf2 expression was determined in total protein extracts by Western blot analysis using an anti-Nrf2 antibody. Anti- $\alpha$-tubulin was used as protein loading control. Results are shown as means of Nrf2/ $\alpha$-Tubulin ratio \pm SEM. Unpaired Student $t$-test; ${ }^{* *} p<0.01 ; n=3$. (B) TNF $\alpha$ amount was measured in the supernatants of THP-1 Nrf2-knockdown cells, treated with compounds 1 , 3 , and 4 at a concentration of 5 $\mu \mathrm{M}$ for $24 \mathrm{~h}$ and then stimulated with $10 \mathrm{ng} / \mathrm{mL}$ LPS for $3 \mathrm{~h}$. The protein secretion of TNF $\alpha$ was determined by ELISA. Data are shown as means of stimulation index

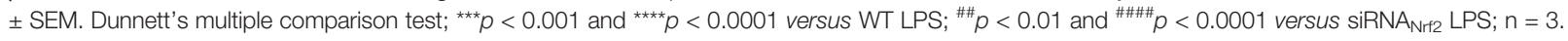

Finally, we investigated the activation of the NF- $\kappa B$ promoter by luciferase assay. To evaluate whether compounds may exert a basal activity on NF- $\kappa$ B promoter, THP-1 cells were transiently transfected with pGL4.32 luciferase reporter construct, containing NF- $\kappa B-$ response elements (RE), and treated with vehicle, $5 \mu \mathrm{M}$ compounds 1-4 and CURC for $24 h$ and, then, analyzed for NF- $\kappa B$ luciferase. No difference in NF- $\kappa \mathrm{B}$ luciferase activity between untreated and treated cells was observed, thus suggesting that compounds did not basally influence NF- $\kappa \mathrm{B}$ pathways (Figure 6C). THP-1 cells were then stimulated with $10 \mathrm{ng} / \mathrm{mL}$ LPS for $6 h$ after treatment with vehicle and $5 \mu \mathrm{M}$ compounds $1-4$ and CURC for $24 h$. As reported in Figure 6D, upon LPS stimulation, NF- $\kappa B$ luciferase activity significantly increased, as expected, and treatments with compounds 2, 3, 4, and CURC significantly reduced it. In accordance with the slight effect on I $\kappa \mathrm{B} \alpha$ phosphorylation and NF$\kappa \mathrm{B}$ nuclear translocation, compound 1 did not hinder the activation of the NF- $\mathrm{KB}$ promoter, thus suggesting that the modulation of cytokine release by this molecule seems not to be driven by NF- $\kappa B$ signaling pathway (Figure 6D).

\section{Differential Regulation of Innate Immune Cytokine Release in Human PBMCs From Healthy Donors}

To further study the differential capability of compounds in modulating cytokine and chemokine release, we moved from THP-1 cells to human primary PBMCs from healthy donors. Human PBMCs were stimulated with $10 \mathrm{ng} / \mathrm{mL}$ LPS for $3 h$ after having been treated with vehicle and $5 \mu \mathrm{M}$ compounds $1-4$ and CURC for $24 h$. The release of a panel of the most common cytokines and chemokines [e.g. IFN $\gamma$, IL-1 $\beta$, IL-4, IL-6, IL-8, IL-12 (p40), IL-12 (p70), IL-13, IL-27, MCP-1, MCP-3, TNF $\alpha$ ] was measured in culture medium by Luminex X-MAP ${ }^{\circledR}$ technology. Protein release of IFN $\gamma$, IL-4, IL-12 (p70), IL-13 and IL-27 was undetectable both in untreated and LPS-stimulated PBMCs from healthy donors, while, exposure of human PBMCs to LPS significantly increased protein release of IL-6,
IL-8, IL-12 (p40), MCP-1, and TNF $\alpha$ compared to controls (Table 2). A differential regulation of cytokine and chemokine release by compounds was observed during immune stimulation. In particular, compounds 1 and CURC, significantly reduced the release of the proinflammatory cytokine IL-6 in LPS-stimulated human PBMCs. In accordance with preliminary results obtained in THP-1 cells, no effect on IL-8 release was observed for 1-4 and CURC, further indicating that all compounds, as well as CURC, did not influence the intracellular pathways regulating IL-8 release. In addition, compounds 1 and 2 significantly decreased IL-12 (p40) release in human PBMCs upon LPS stimulation (Table 2). No differences in IL- $1 \beta$ and MCP-3 release were observed between PBMCs that were stimulated by LPS, untreated, or treated with compounds or CURC PBMCs (Table 2). Interestingly, compounds 1, 2, and CURC were capable to significantly attenuate the release of the chemokine MCP-1 in LPS-stimulated PBMCs from healthy patients. In contrast, compounds 3 and 4 did not affect MCP-1 release, revealing the same activity trend observed for Nrf2 induction (Table 2). Notably, such results are consistent with evidence from literature reporting that, after innate immune stimulation, treatment of human PBMCs with Nrf2 activators, such as the Nrf2 agonist CDDO-Me (bardoxolone methyl), markedly reduced LPS-evoked MCP-1/ CCL2 production and that this effect was not specific to LPSinduced immune responses, as Nrf2 activation also reduced MCP1/CCL2 production after stimulation with IL-6 (Eitas et al., 2017). Furthermore, compound 4 and CURC confirmed their capability to significantly reduce TNF $\alpha$ release in LPS-stimulated PBMCs, as previously observed in the THP-1 cell line (Table 2).

\section{DISCUSSION}

The transcription factor Nrf2 regulates a complex network of cellular responses to oxidative stress and inflammation. Cysteine residues of its repressor Keap1 act as sensor sites for Nrf2 

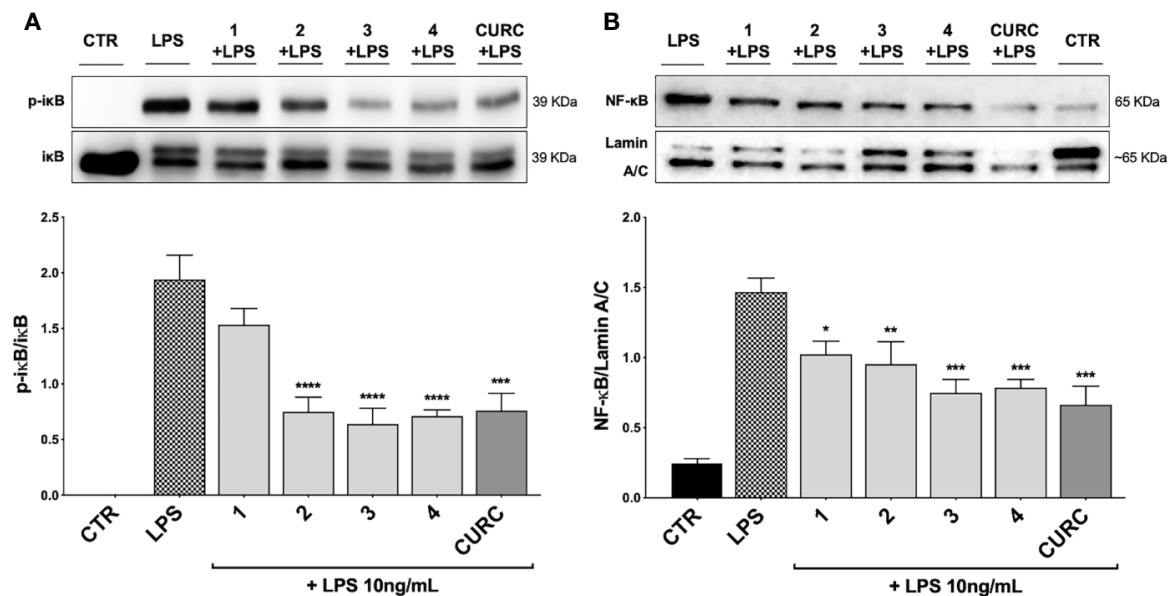

C

D
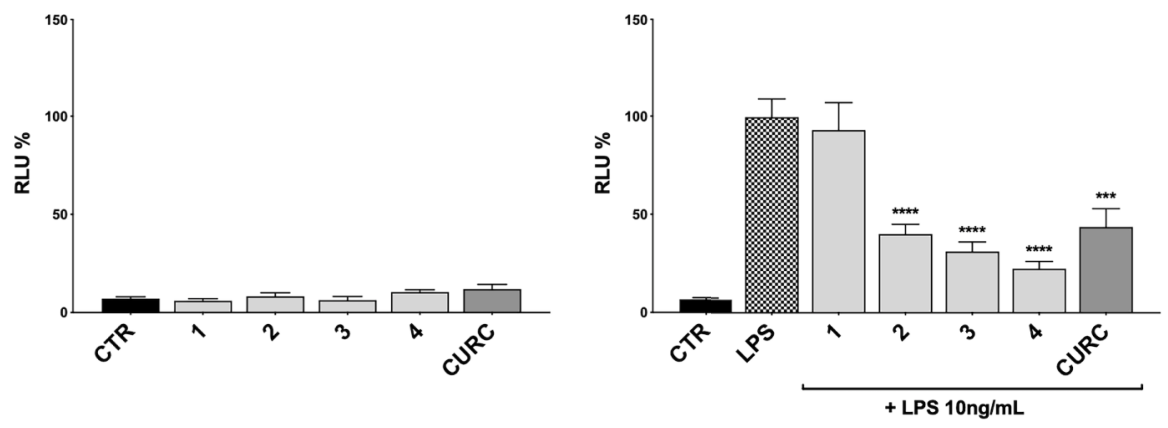

FIGURE 6 | Modulation of NF-KB pathway by compounds and CURC in LPS-stimulated THP-1 cells. (A) THP-1 cells were treated with $5 \mu$ M compounds $1-4$ and CURC for $24 \mathrm{~h}$ and then stimulated with $10 \mathrm{ng} / \mathrm{mL}$ LPS for $45 \mathrm{~min}$. After stimulation, $\mathrm{p}$-I $\mathrm{kB} \alpha$ expression was determined in total protein extracts by Western blot

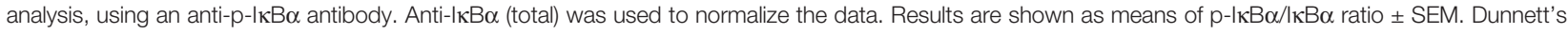
multiple comparison test; ${ }^{* \star *} p<0.001$ and ${ }^{* \star * *} p<0.0001$ versus LPS; $n=5$. (B) THP-1 cells were treated for $24 \mathrm{~h}$ with compounds $1-4$ and CURC at a concentration of $5 \mu \mathrm{M}$ and then stimulated with $10 \mathrm{ng} / \mathrm{mL}$ LPS for $90 \mathrm{~min}$. After isolation, nuclear extracts were examined by Western blot analysis and NF-kB expression was determined using an anti-NF-kB antibody. Anti-lamin A/C was used as protein loading control. Results are shown as means of NF-kB/Lamin A/C ratio \pm SEM. Dunnett's multiple comparison test; ${ }^{*} p<0.05,{ }^{* *} p<0.01$ and ${ }^{* * *} p<0.001$ versus LPS; $n=5$. (C, D) THP-1 cells were transiently transfected with pGL4.32 [luc2P/NF-KB-RE/Hygro] Vector reporter construct, and subsequently treated with compounds $1-4$ and CURC at a concentration of $5 \mu \mathrm{M}$ for $24 \mathrm{~h}$. After treatments, the cells were stimulated (D) or not (C) with $10 \mathrm{ng} / \mathrm{mL}$ LPS for $6 \mathrm{~h}$. For each condition, luciferase activity was expressed as RLU\% and compared to CTR values assumed at 100\%. Results are shown as means \pm SEM. Dunnett's multiple comparison test; ${ }^{* \star *} p<0.001$ and ${ }^{* \star \star *} p<0.0001$ versus LPS; $n=3$.

TABLE 2 | Differential regulation of innate immune cytokine release in human PBMCs from healthy donors.

\begin{tabular}{|c|c|c|c|c|c|c|c|}
\hline \multicolumn{8}{|c|}{ Luminex xMAP ${ }^{\circledR}$ Technology } \\
\hline & CTR & LPS10 ng/mL & $1+$ LPS & $2+$ LPS & $3+$ LPS & 4+ LPS & CURC+ LPS \\
\hline $\mathrm{IFN \gamma}$ & $\mathrm{Nd}$ & $\mathrm{Nd}$ & $\mathrm{Nd}$ & $\mathrm{Nd}$ & $\mathrm{Nd}$ & nd & nd \\
\hline IL-4 & $\mathrm{Nd}$ & $\mathrm{Nd}$ & nd & $\mathrm{Nd}$ & $\mathrm{Nd}$ & nd & nd \\
\hline IL-6 & $33.20 \pm 6.38^{\star \star \star \star}$ & $164.4 \pm 12.1$ & $129.2 \pm 7.68^{\star}$ & $139.9 \pm 8.32$ & $147.1 \pm 10.5$ & $156.0 \pm 11.4$ & $99.65 \pm 6.71^{* \star \star *}$ \\
\hline IL-8 & $1659 \pm 178^{\star \star \star \star *}$ & $3349 \pm 292$ & $4012 \pm 141$ & $3272 \pm 224$ & $2794 \pm 124$ & $3026 \pm 333$ & $2665 \pm 221$ \\
\hline IL-12 (p40) & $2.44 \pm 0.14^{\star *}$ & $5.56 \pm 0.57$ & $2.40 \pm 0.12^{\star *}$ & $3.28 \pm 0.27^{\star}$ & $4.82 \pm 0.87$ & $5.30 \pm 0.90$ & $3.61 \pm 0.70$ \\
\hline IL-27 & nd & $\mathrm{Nd}$ & nd & nd & nd & nd & nd \\
\hline MCP-1 & $1149 \pm 89.2^{*}$ & $1627 \pm 139$ & $899 \pm 163^{\star \star \star}$ & $819 \pm 89.5^{\star \star \star \star}$ & $1243 \pm 125$ & $1198 \pm 105$ & $625 \pm 76.9^{\star \star \star \star *}$ \\
\hline MCP-3 & $38.9 \pm 8.2$ & $50.6 \pm 8.9$ & $36.7 \pm 6.0$ & $35.11 \pm 5.7$ & $43.0 \pm 8.3$ & $42.1 \pm 6.9$ & $25.3 \pm 3.3$ \\
\hline TNF $\alpha$ & $13.75 \pm 2.76^{\star \star \star \star}$ & $350.2 \pm 18.6$ & $264.8 \pm 6.74$ & $295.2 \pm 18.7$ & $328.3 \pm 16.3$ & $220.8 \pm 31.1^{\star *}$ & $252.8 \pm 38.6^{*}$ \\
\hline
\end{tabular}

PBMCs were treated with compounds $1-4$ and CURC at a concentration of $5 \mu M$ for $24 \mathrm{~h}$, and then stimulated with $10 \mathrm{ng} / \mathrm{mL} L \mathrm{LPS}$ for $3 \mathrm{~h}$. IFN $\gamma$, IL-1 $\mathrm{k}, \mathrm{LL}-4$, IL-6, IL-8, IL-12 (p40), IL-12 (p70), IL-13, IL-27, MCP-1, MCP-3, TNF $\alpha$ protein release was measured in PBMC supernatants by Luminex XMAP ${ }^{\circledR}$ Technology. Data are presented as means of released picograms per $\mathrm{mL}(\mathrm{pg} / \mathrm{mL}) \pm$ SEM. Dunnett's multiple comparison test; ${ }^{*} p<0.05 ;{ }^{* *} p<0.01 ;{ }^{* * *} p<0.001$ and ${ }^{* * * *} p<0.0001$ versus LPS; $n=5$. 
electrophilic activators. Thus, we studied a set of previously synthesized compounds (1,2, and 3), for which the ability to induce the Nrf2 pathway was strictly related to the (pro)electrophilic character of the molecule in THP-1 cells, a widely used cellular model for the immune modulation approach (Chanput et al., 2014). In agreement with previous results (Simoni et al., 2017; Serafini et al., 2019; Catanzaro et al., 2020), a significant effect was detected for the Nrf2 inducers 1 and 2, carrying a catechol moiety and/or an $\alpha, \beta$-unsaturated carbonyl group, while no effect was observed for compound 3 , lacking both (pro)-electrophilic features (Table 1). The same lack of effect was observed for compound 4, which was included in the study to exclude possible oxidative activation into electrophilic metabolites such as quinone methide, which could provide an additional site for adduct formation.

Based on these results, we investigated the potential effects of the compounds on the secretion of pro-inflammatory cytokines upon immune stimulation (e.g. LPS from E. coli) in the same cellular model. We found that both compounds which induced Nrf2 (1 and 2) as well as compounds inactive on the Nrf2 pathway (3 and 4) were capable to attenuate the release of the pro-inflammatory cytokines TNF $\alpha$ (Figure 3A) and IL-1 $\beta$ (Figure 4B), but not IL-8 secretion (Figure 3B), thus suggesting that the reduction of cytokine release by compounds could not be directly ascribed to the activation of Nrf2 pathway. Accordingly, the ability of compounds to attenuate the secretion of TNF $\alpha$, upon immune stimulation, was also observed after siRNA mediated Nrf2 knockdown (Figure 5). To further dissect the molecular mechanism underlying the reduction of cytokine release induced by compounds $1-4$, we investigated their potential interplay with other signaling cascades, specifically focusing on the NF- $\kappa \mathrm{B}$ pathway, a pivotal mediator of inflammatory responses and critical regulator of multiple aspects of innate and adaptative immune functions (Häcker and Karin, 2006; Perkins, 2007). All compounds, with the exception of compound 1 ,

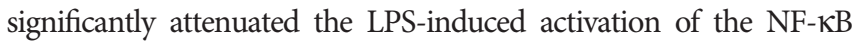
canonical pathway, by impairing the upstream phosphorylation of
$\mathrm{I} \kappa \mathrm{B} \alpha, \mathrm{NF}-\kappa \mathrm{B}$ nuclear translocation, as well as the activation of the $\mathrm{NF}-\kappa \mathrm{B}$ promoter (Figure 5). As a consequence, the ability of compounds 2, 3, and 4 to reduce the activation of NF- $\kappa B$ pathway may account, at least in part, for their observed effect on proinflammatory cytokine release. Notably, both Nrf2 and NF- $\mathrm{KB}$ offer unique patterns of thiol modifications, indicating electrophilic signaling mediators as a valuable instrument to control their redox-sensitive transcriptional regulatory function. However, while a (pro)-electrophilic feature is required for Nrf2 induction, suggesting covalent adduction as the triggering event, both (pro)electrophile 2 and non-electrophilic compounds 3 and 4 were able to inhibit NF- $\kappa$ B activation, revealing a different mode of interaction (Figure 7). Noteworthy, compound 1, carrying both the catechol moiety and the $\alpha, \beta$-unsaturated carbonyl group, was unable to significantly modulate the NF- $\kappa \mathrm{B}$ pathway. The modulation of cytokine release by this molecule might be, at least in part, related to anti-inflammatory effect mediated by the induction of $\mathrm{Nrf} 2$ targets, such as HO-1 (Roach et al., 2009). Accordingly, HO-1 expression has been demonstrated to decrease the LPS-stimulated secretion of cytokines and chemokines such as MCP-1, IL-6, IL-10, and TNF $\alpha$ in murine and human macrophages (Roach et al., 2009). Altogether, these results indicate that an electrophilic moiety is neither necessary nor per se sufficient to guarantee inhibition of the pro-inflammatory transcriptional activity of NF$\kappa \mathrm{B}$, with shape complementarity emerging as a plausible feature of target recognition. The different biological behavior of electrophiles 1 and 2, which only varies in the presence or absence of two double bonds, might indeed reflect the more constrained conformation assumed by compound 1 with respect to flexible compound 2 . The conjugation extended throughout most of the backbone stabilized compound 1 in a planar conformation, as opposed to the sp3 counterparts which manifested a maximum in energy for the same state (Supplementary Figure 2). Moreover, the possibility for compounds 2-4 to populate several conformations due to lower energy barriers might indicate that a conformational selection or induced fit effect in the ligand is necessary to execute the desired

\section{Nrf2 activation}

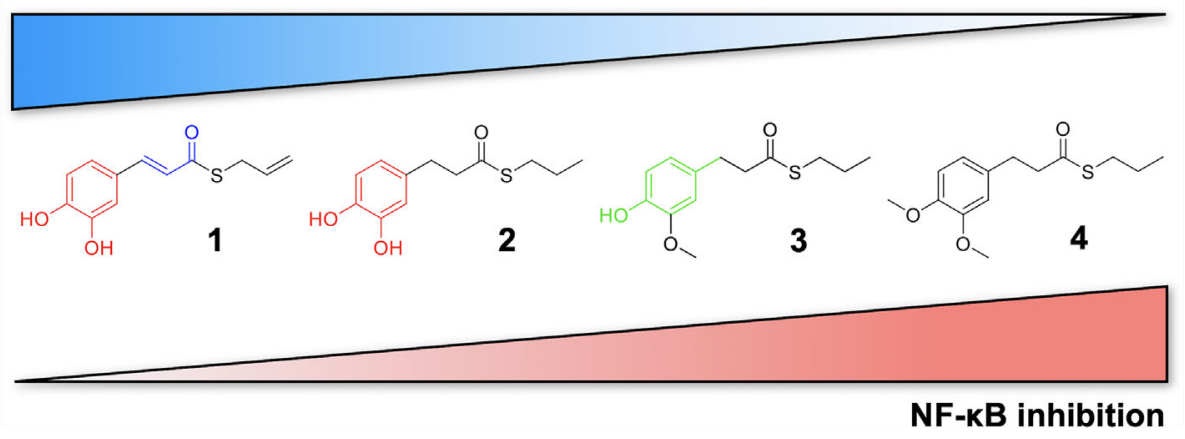

FIGURE 7 | Differential modulation of Nrf2 and NF-kB intracellular signaling pathways by compounds. Electrophile 1, carrying both the catechol moiety (red) and the $\alpha, \beta$-unsaturated carbonyl group (blue), is the most active Nrf2 inducer, while being devoid of activity on NF-kB pathway. Conversely, the non-electrophilic compound 4 , synthesized to exclude eventual oxidative transformation of the methoxyphenol ring (green) of 3 into reactive metabolites, is the most potent NF-kB inhibitor, with no impact on Nrf2 activation. 
activity. The overall effects of compounds 1-4 on Nrf2 and NF- $\kappa B$ intracellular pathways were summarized in Figure 8.

When moving in a human primary model, by Luminex XMAP $^{\circledR}$ technology, we screened the effects of compounds on a panel of cytokines and chemokines (e.g. IFN $\gamma$, IL-1 $\beta$, IL-4, IL-6, IL-8, IL-12 (p40), IL-12 (p70), IL-13, IL-27, MCP-1, MCP-3, $\mathrm{TNF} \alpha$ ) in order to unveil their potential modulatory effect on other inflammatory mediators (Table 2). Compared to data observed in THP-1 cells, we found a differential regulation of innate immune cytokine production by compounds, in line with previous data (Schildberger et al., 2013). In particular, compounds 1 and CURC significantly reduced the secretion of the pro-inflammatory cytokine IL-6 in LPS-stimulated human PBMCs, while compound 4 and CURC attenuated TNF $\alpha$ release (Table 2). Furthermore, compounds acting as Nrf2 inducers (1 and 2) also suppressed the secretion of IL-12 (p40), corroborating the hypothesis that inhibition of IL-12 expression may be mediated by Nrf2 activation, as suggested by Macoch et al. (2015). Consistently, tert-butylhydroquinone, a well-known Nrf2 inducer, has been reported to activate Nrf2 and to inhibit the induction of IL-12 expression by LPS (Macoch et al., 2015). However, further investigations are required to unravel the molecular mechanism by which Nrf2 represses IL-12 production and secretion.

In human PBMCs, we further found that only compounds acting as Nrf2 inducers (1 and 2) significantly suppressed the release of MCP-1, after LPS stimulation (Table 2). In accordance with data from the literature (Eitas et al., 2017), this result indicates that $\mathrm{MCP}-1$ production may rely on activation of the transcription factor Nrf2. Thus, the effect of Nrf2 inducers on MCP-1/CCL2 suggests a novel aspect of Nrf2 pharmacological activation as a regulator of key immunomodulatory functions. This finding represents a potentially generalizable aspect of pharmacological Nrf2 activation occurring with different stimuli (e.g. LPS, IL-6) and consistent across more than 60 individual human samples, as reported by Eitas et al. (2017). Thus, contrary to the prevalent view that Nrf2 represses inflammatory processes through redox control, we demonstrated that Nrf2 activation also directly counteracts the production of a key chemokine, by possibly regulating the expression of its encoding gene. Such hypothesis is consistent with data reporting Nrf2-mediated downregulation of proinflammatory mediator gene expression (Kobayashi et al., 2016). However, the precise molecular mechanism underlying Nrf2 and MCP-1 crosstalk is still elusive. Interestingly, by regulating the production of the chemokine MCP-1, Nrf2 can be considered an upstream regulator of MCP-1 production, thereby providing a molecular basis for a Nrf2-mediated anti-inflammatory approach. In this regard, elevated systemic MCP-1 system levels have been linked to worse outcomes in patients with cardiovascular disease (Martín-Ventura et al., 2009), and pulmonary accumulation of MCP-1 has been reported in patients with acute respiratory distress syndrome (Rosseau et al., 2000). Hence, targeting transcriptional accumulation of MCP-1 through pharmacological Nrf2 activation may represent a promising therapeutic approach.

Although the THP-1 and human PBMCs response can hint to potential responses that may occur in vivo, these results need to be validated in in vivo studies to draw more definite conclusions. Moreover, further mechanistic investigations are required to

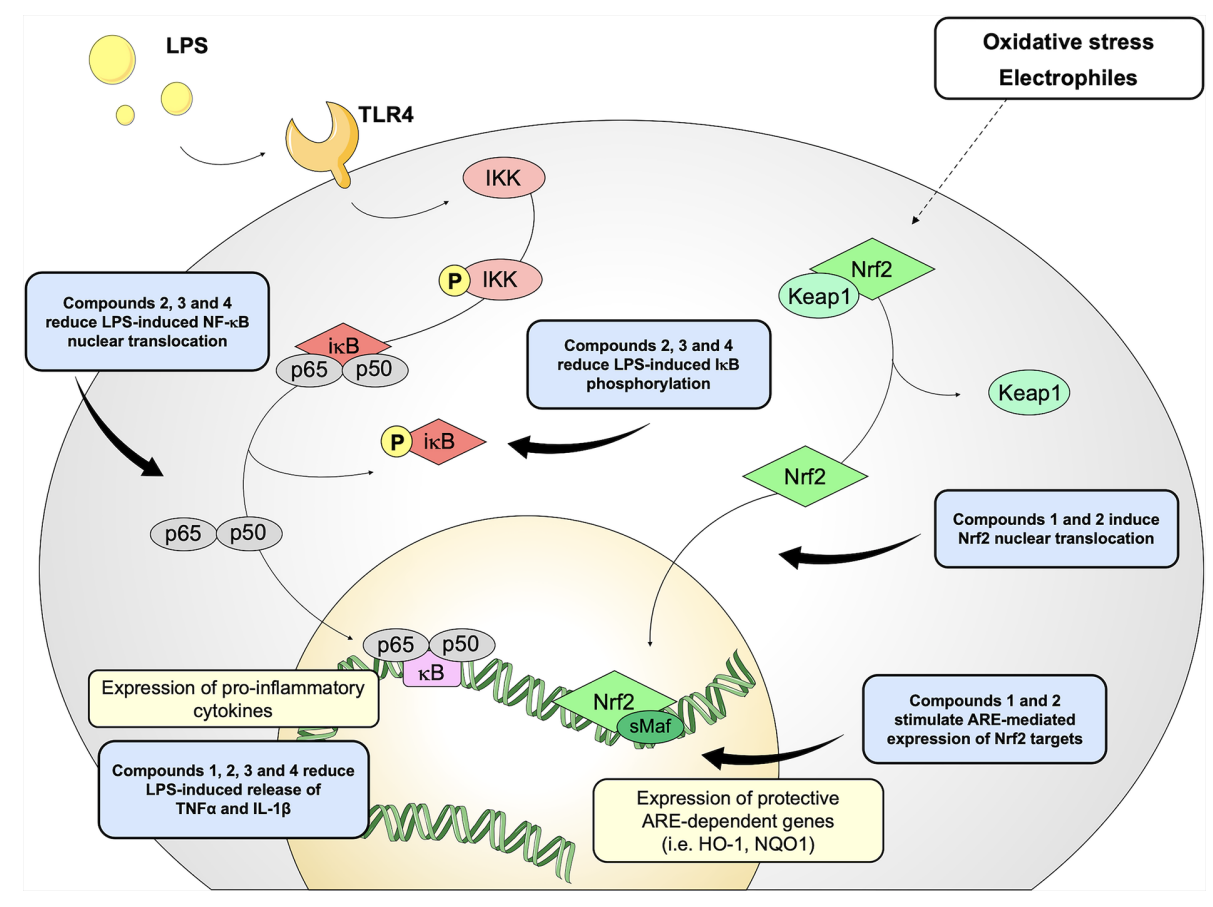

FIGURE 8 | Schematic representation of the effects induced by compounds 1-4 on Nrf2 and NF-кB pathways. 
unravel the biological connection among Nrf2 activation, innate immune cytokine production, and the regulation of the NF$\kappa \mathrm{B}$ pathway.

\section{DATA AVAILABILITY STATEMENT}

The raw data supporting the conclusions of this article will be made available by the authors, without undue reservation, to any qualified researcher.

\section{ETHICS STATEMENT}

The studies involving human participants were reviewed and approved by Ethical Committee Project approval: Fulop_20192877. The patients/participants provided their written informed consent to participate in this study.

\section{AUTHOR CONTRIBUTIONS}

Conceived and designed the experiments: FF, MC, EC, MRo, and CL. Chemical synthesis: FB and MRo. Performed the

\section{REFERENCES}

Baeuerle, P. A., and Baltimore, D. (1996). NF-kappa B: ten years after. Cell 87, $13-$ 20. doi: 10.1016/s0092-8674(00)81318-5

Basagni, F., Lanni, C., Minarini, A., and Rosini, M. (2019). Lights and shadows of electrophile signaling: focus on the Nrf2-Keap1 pathway. Future Med. Chem. 11, 707-721. doi: 10.4155/fmc-2018-0423

Binkley, J. S., Pople, J. A., and Hehre, W. J. (1980). Self-consistent molecular orbital methods. 21. Small split-valence basis sets for first-row elements. J. Am. Chem. Soc 102, 939-947. doi: 10.1021/ja00523a008

Buoso, E., Ronfani, M., Galasso, M., Ventura, D., Corsini, E., and Racchi, M. (2019). Cortisol-induced SRSF3 expression promotes GR splicing, RACK1 expression and breast cancer cells migration. Pharmacol. Res. 143, 17-26. doi: 10.1016/ j.phrs.2019.03.008

Catanzaro, M., Lanni, C., Basagni, F., Rosini, M., Govoni, S., and Amadio, M. (2020). Eye-Light on Age-Related Macular Degeneration: Targeting Nrf2Pathway as a Novel Therapeutic Strategy for Retinal Pigment Epithelium. Front. Pharmacol. 11, 844. doi: 10.3389/fphar.2020.00844

Chanput, W., Mes, J. J., and Wichers, H. J. (2014). THP-1 cell line: an in vitro cell model for immune modulation approach. Int. Immunopharmacol. 23, 37-45. doi: 10.1016/j.intimp.2014.08.002

D’amico, R., Fusco, R., Gugliandolo, E., Cordaro, M., Siracusa, R., Impellizzeri, D., et al. (2019). Effects of a new compound containing Palmitoylethanolamide and Baicalein in myocardial ischaemia/reperfusion injury in vivo. Phytomed. Int. J. Phytother. Phytopharm. 54, 27-42. doi: 10.1016/j.phymed.2018.09.191

Eitas, T. K., Stepp, W. H., Sjeklocha, L., Long, C. V., Riley, C., Callahan, J., et al. (2017). Differential regulation of innate immune cytokine production through pharmacological activation of Nuclear Factor-Erythroid-2-Related Factor 2 (NRF2) in burn patient immune cells and monocytes. PLoS One 12, e0184164. doi: 10.1371/journal.pone.0184164

Fusco, R., Gugliandolo, E., Biundo, F., Campolo, M., Di Paola, R., and Cuzzocrea, S. (2017). Inhibition of inflammasome activation improves lung acute injury induced by carrageenan in a mouse model of pleurisy. FASEB J. 31, 34973511. doi: 10.1096/fj.201601349R

Gomes, A., Capela, J. P., Ribeiro, D., Freitas, M., Silva, A. M. S., Pinto, D. C. G. A., et al. (2015). Inhibition of NF-kB activation and cytokines production in THP- experiments and analyzed the data: FF, MC, and EB. Quantum mechanics data: DM and SR. Critical discussion: FF, MC, EC, MRa, MA, TF, SG, MRo, and CL. Funding acquisition: EF, TP, EC, MRa, MRo, and CL.

\section{FUNDING}

Research has been supported by the University of Pavia (grants from FR\&G 2019, Fondo Ricerca \& Giovani, to CL; PRIN 2017B9NCSX_003 to MRa; an Educational Grant from Aboca S.p.A. to MRa and SG), the University of Milan (grants from PRIN 2017MLC3NF to EC), the University of Bologna (grants from the RFO to MRo) and grants from Université de Sherbrooke, FRQS "AUDACE" program and the Research Center of the CHUS to EF and TF).

\section{SUPPLEMENTARY MATERIAL}

The Supplementary Material for this article can be found online at: https://www.frontiersin.org/articles/10.3389/fphar.2020. 01256/full\#supplementary-material

1 monocytes by 2-styrylchromones. Med. Chem. Shariqah United Arab Emir. 11, 560-566. doi: 10.2174/1573406411666150209114702

Häcker, H., and Karin, M. (2006). Regulation and function of IKK and IKK-related kinases. Sci. STKE Signal Transduction Knowl. Environ. 2006, re13. doi: 10.1126/ stke.3572006re13

Hayden, M. S., West, A. P., and Ghosh, S. (2006). NF- $\kappa$ B and the immune response. Oncogene 25, 6758-6780. doi: 10.1038/sj.onc.1209943

Hayes, J. D., and Dinkova-Kostova, A. T. (2014). The Nrf2 regulatory network provides an interface between redox and intermediary metabolism. Trends Biochem. Sci. 39, 199-218. doi: 10.1016/j.tibs.2014.02.002

Iizuka, T., Ishii, Y., Itoh, K., Kiwamoto, T., Kimura, T., Matsuno, Y., et al. (2005). Nrf2-deficient mice are highly susceptible to cigarette smoke-induced emphysema. Genes Cells Devoted Mol. Cell. Mech. 10, 1113-1125. doi: 10.1111/ j.1365-2443.2005.00905.x

Innamorato, N. G., Rojo, A.II, García-Yagüe, A. J., Yamamoto, M., de Ceballos, M. L., and Cuadrado, A. (2008). The transcription factor Nrf2 is a therapeutic target against brain inflammation. J. Immunol. Baltim. Md 1950 181, 680-689. doi: 10.4049/jimmunol.181.1.680

Ishii, Y., Itoh, K., Morishima, Y., Kimura, T., Kiwamoto, T., Iizuka, T., et al. (2005). Transcription factor Nrf2 plays a pivotal role in protection against elastaseinduced pulmonary inflammation and emphysema. J. Immunol. Baltim. Md 1950 175, 6968-6975. doi: 10.4049/jimmunol.175.10.6968

Kastrati, I., Siklos, M.II, Calderon-Gierszal, E. L., El-Shennawy, L., Georgieva, G., Thayer, E. N., et al. (2016). Dimethyl Fumarate Inhibits the Nuclear Factor $\kappa B$ Pathway in Breast Cancer Cells by Covalent Modification of p65 Protein. J. Biol. Chem. 291, 3639-3647. doi: 10.1074/jbc.M115.679704

Knatko, E. V., Ibbotson, S. H., Zhang, Y., Higgins, M., Fahey, J. W., Talalay, P., et al. (2015). Nrf2 Activation Protects against Solar-Simulated Ultraviolet Radiation in Mice and Humans. Cancer Prev. Res. Phila. Pa 8, 475-486. doi: 10.1158/1940-6207.CAPR-14-0362

Kobayashi, M., and Yamamoto, M. (2006). Nrf2-Keap1 regulation of cellular defense mechanisms against electrophiles and reactive oxygen species. Adv. Enzyme Regul. 46, 113-140. doi: 10.1016/j.advenzreg.2006.01.007

Kobayashi, E. H., Suzuki, T., Funayama, R., Nagashima, T., Hayashi, M., Sekine, H., et al. (2016). Nrf2 suppresses macrophage inflammatory response by blocking proinflammatory cytokine transcription. Nat. Commun. 7:11624. doi: 10.1038/ ncomms11624 
Kohn, W., and Sham, L. J. (1965). Self-Consistent Equations Including Exchange and Correlation Effects. Phys. Rev. 140, A1133-A1138. doi: 10.1103/PhysRev.140.A1133

Kumar, P., Nagarajan, A., and Uchil, P. D. (2018). Analysis of Cell Viability by the MTT Assay. Cold Spring Harb. Protoc. 2018. doi: 10.1101/pdb.prot095505

Le Page, A., Garneau, H., Dupuis, G., Frost, E. H., Larbi, A., Witkowski, J. M., et al. (2017). Differential Phenotypes of Myeloid-Derived Suppressor and T Regulatory Cells and Cytokine Levels in Amnestic Mild Cognitive Impairment Subjects Compared to Mild Alzheimer Diseased Patients. Front. Immunol. 8:783. doi: 10.3389/fimmu.2017.00783

Luis, P. B., Boeglin, W. E., and Schneider, C. (2018). Thiol Reactivity of Curcumin and Its Oxidation Products. Chem. Res. Toxicol. 31, 269-276. doi: 10.1021/ acs.chemrestox.7b00326

Macoch, M., Morzadec, C., Génard, R., Pallardy, M., Kerdine-Römer, S., Fardel, O., et al. (2015). Nrf2-dependent repression of interleukin-12 expression in human dendritic cells exposed to inorganic arsenic. Free Radic. Biol. Med. 88, 381-390. doi: 10.1016/j.freeradbiomed.2015.02.003

Martín-Ventura, J. L., Blanco-Colio, L. M., Tuñón, J., Muñoz-García, B., MadrigalMatute, J., Moreno, J. A., et al. (2009). Biomarkers in cardiovascular medicine. Rev. Esp. Cardiol. 62, 677-688. doi: 10.1016/s1885-5857(09)72232-7

Møller, C., and Plesset, M. S. (1934). Note on an Approximation Treatment for Many-Electron Systems. Phys. Rev. 46, 618-622. doi: 10.1103/PhysRev.46.618

Niture, S. K., Khatri, R., and Jaiswal, A. K. (2014). Regulation of Nrf2-an update. Free Radic. Biol. Med. 66, 36-44. doi: 10.1016/j.freeradbiomed.2013.02.008

Pawelec, G., Ferguson, F. G., and Wikby, A. (2001). The SENIEUR protocol after 16 years. Mech. Ageing Dev. 122, 132-134. doi: 10.1016/s0047-6374(00)00240-2

Perkins, N. D. (2007). Integrating cell-signalling pathways with NF-kappaB and IKK function. Nat. Rev. Mol. Cell Biol. 8, 49-62. doi: 10.1038/nrm2083

Petersson, G. A., and Al-Laham, M. A. (1991). A complete basis set model chemistry. II. Open-shell systems and the total energies of the first-row atoms. J. Chem. Phys. 94, 6081-6090. doi: 10.1063/1.460447

Quinti, L., Dayalan Naidu, S., Träger, U., Chen, X., Kegel-Gleason, K., Llères, D., et al. (2017). KEAP1-modifying small molecule reveals muted NRF2 signaling responses in neural stem cells from Huntington's disease patients. Proc. Natl. Acad. Sci. U. S. A. 114, E4676-E4685. doi: 10.1073/pnas.1614943114

Roach, J. P., Moore, E. E., Partrick, D. A., Damle, S. S., Silliman, C. C., McIntyre, R. C., et al. (2009). Heme oxygenase-1 induction in macrophages by a hemoglobin-based oxygen carrier reduces endotoxin-stimulated cytokine secretion. Shock Augusta Ga 31, 251-257. doi: 10.1097/SHK.0b013e3181834115

Rosseau, S., Hammerl, P., Maus, U., Walmrath, H. D., Schütte, H., Grimminger, F., et al. (2000). Phenotypic characterization of alveolar monocyte recruitment in acute respiratory distress syndrome. Am. J. Physiol. Lung Cell. Mol. Physiol. 279, L25-L35. doi: 10.1152/ajplung.2000.279.1.L25

Sakai, J., Cammarota, E., Wright, J. A., Cicuta, P., Gottschalk, R. A., Li, N., et al. (2017). Lipopolysaccharide-induced NF- $\kappa B$ nuclear translocation is primarily dependent on MyD88, but TNF $\alpha$ expression requires TRIF and MyD88. Sci. Rep. 7, 1428. doi: 10.1038/s41598-017-01600-y
Satoh, T., McKercher, S. R., and Lipton, S. A. (2013). Nrf2/ARE-mediated antioxidant actions of pro-electrophilic drugs. Free Radic. Biol. Med. 65, 645-657. doi: 10.1016/j.freeradbiomed.2013.07.022

Schildberger, A., Rossmanith, E., Eichhorn, T., Strassl, K., and Weber, V. (2013). Monocytes, peripheral blood mononuclear cells, and THP-1 cells exhibit different cytokine expression patterns following stimulation with lipopolysaccharide. Mediators Inflamm. 2013, 697972. doi: 10.1155/2013/ 697972

Serafini, M. M., Catanzaro, M., Fagiani, F., Simoni, E., Caporaso, R., Dacrema, M., et al. (2019). Modulation of Keap1/Nrf2/ARE Signaling Pathway by Curcumaand Garlic-Derived Hybrids. Front. Pharmacol. 10, 1597. doi: 10.3389/ fphar.2019.01597

Simoni, E., Serafini, M. M., Bartolini, M., Caporaso, R., Pinto, A., Necchi, D., et al. (2016). Nature-Inspired Multifunctional Ligands: Focusing on Amyloid-Based Molecular Mechanisms of Alzheimer's Disease. ChemMedChem 11, 13091317. doi: $10.1002 / \mathrm{cmdc} .201500422$

Simoni, E., Serafini, M. M., Caporaso, R., Marchetti, C., Racchi, M., Minarini, A., et al. (2017). Targeting the Nrf2/Amyloid-Beta Liaison in Alzheimer's Disease: A Rational Approach. ACS Chem. Neurosci. 8, 1618-1627. doi: 10.1021/ acschemneuro.7b00100

Tanigawa, S., Fujii, M., and Hou, D.-X. (2007). Action of Nrf2 and Keap1 in AREmediated NQO1 expression by quercetin. Free Radic. Biol. Med. 42, 16901703. doi: 10.1016/j.freeradbiomed.2007.02.017

Thimmulappa, R. K., Lee, H., Rangasamy, T., Reddy, S. P., Yamamoto, M., Kensler, T. W., et al. (2006). Nrf2 is a critical regulator of the innate immune response and survival during experimental sepsis. J. Clin. Invest. 116, 984-995. doi: 10.1172/JCI25790

Wardyn, J. D., Ponsford, A. H., and Sanderson, C. M. (2015). Dissecting molecular cross-talk between Nrf2 and NF- $\mathrm{KB}$ response pathways. Biochem. Soc Trans. 43, 621-626. doi: 10.1042/BST20150014

Woodcock, C.-S. C., Huang, Y., Woodcock, S. R., Salvatore, S. R., Singh, B., GolinBisello, F., et al. (2018). Nitro-fatty acid inhibition of triple-negative breast cancer cell viability, migration, invasion, and tumor growth. J. Biol. Chem. 293, 1120-1137. doi: 10.1074/jbc.M117.814368

Conflict of Interest: The authors declare that the research was conducted in the absence of any commercial or financial relationships that could be construed as a potential conflict of interest.

Copyright (c) 2020 Fagiani, Catanzaro, Buoso, Basagni, Di Marino, Raniolo, Amadio, Frost, Corsini, Racchi, Fulop, Govoni, Rosini and Lanni. This is an open-access article distributed under the terms of the Creative Commons Attribution License (CC BY). The use, distribution or reproduction in other forums is permitted, provided the original author(s) and the copyright owner(s) are credited and that the original publication in this journal is cited, in accordance with accepted academic practice. No use, distribution or reproduction is permitted which does not comply with these terms. 